\title{
Fixed-Mix Rules in an Evolutionary Market Using a Factor Model for Dividends
}

\author{
Konstantinos Mavroudis* and Craig Nolder ${ }^{\dagger}$
}

\begin{abstract}
In this paper we explore the impact of various constant-proportions investment strategies (or FixedMix Rules) in an economic evolutionary market. Dividends are generated according to a new Dividend Factor Model. Furthermore, Dividends were estimated and calibrated from data using Principal Component Analysis. Moreover, we perform simulations to study the long-run outcome of an evolutionary competition with several well diversified constant-proportions strategies, among them some innovative strategies. We present and compare a variety of simulations with dividends being artificially generated according to our Dividend Model. Our simulation results are important for both theoretical and practical reasons. In theoretical terms we have a model where, although the true rational strategy is the only probable dominant strategy, it is also possible for some "behavioral" rules to perform better under specific circumstances. In practical terms we suggest new constant-proportions strategies that could be superior for investors at least in the short run.
\end{abstract}

Keywords: Dividend Factor Model, Principal Components Analysis, Constant Proportions Strategies, Evolutionary Portfolio Theory

\section{Introduction}

The objective of this paper is to study the impact of constant-proportions investment strategies on investor's wealth in the context of an Evolutionary Market Model given a new Factor Model for dividends. Toward that end, we employ new constant-proportions strategies in relation to an Evolutionary Finance framework given our particular Dividend Factor Model. We believe that the synthesis of these concepts not only explains some peculiar financial phenomena but also provides under specific circumstances several profitable opportunities for investors.

This objective requires the intersection of three atypical notions of mathematical finance. The first notion involves the use of constant-proportions investment strategies (alternatively called fixed-mix trading rules or rebalancing rules) that try to exploit concepts such as volatility pumping or excess growth in order to explain financial growth. The second involves the recently discovered framework of Evolutionary Finance that explains financial phenomena under a Darwinian paradigm. That is, it exploits concepts such as mutation and selection in order to study how trading strategies and financial growth evolve over time. The last notion involves the construction of a new model that connects dividends with macroeconomic variables utilizing familiar concepts and techniques from multivariate analysis. In that way, we believe that we not only can explain complicated financial phenomena but also provide a new framework for subsequent analysis. In addition, under particular circumstances, our results suggest profitable strategies that are readily available to investors.

Although the idea of constant-proportions investment strategies is not prominent in financial theory, many argue that these strategies could provide superior profits to investors (Fernholz and Maguire [12]). So far, a general model for the mechanisms of these investment strategies does not exist although there are some examples in the literature such as volatility pumping (Luenberger [19]) and excess growth (Fernholz [11]) that connect these rules with a generated price volatility. Moreover, recently some theoretical results have established that, when asset returns are stationary and ergodic, fixed-mix trading rules generate a portfolio

\footnotetext{
*Department of Mathematics, Florida State University, Tallahassee, FL 32306-4510, USA, kmavroud@math.fsu.edu.

${ }^{\dagger}$ Department of Mathematics, Florida State University, Tallahassee, FL 32306-4510, USA, nolder@math.fsu.edu.
} 
growth rate in excess of the individual asset growth rates (Dempster, et. al. [7]). The intuition behind this mechanism is that such strategies implicitly follow a buy-low/sell-high rationale, although which price could be considered as high or low is an arbitrary concept. Hence, if market prices fluctuate, a rebalancing rule has to adjust the number of shares in order to keep constant the proportions of wealth allocated to predetermined stocks.

Evolutionary Finance is based initially on the idea of evolutionary game theory that was formulated in the context of biology by Maynard Smith and Price ([23, 24]). Evolutionary Finance utilizes the idea that the portfolio rule (or strategy) that eventually dominates the market becomes the best strategy (response) for all market participants. Thus, the equilibrium condition is not static but arises from the interaction of many strategies through an evolutionary process. Evolutionary Finance is not totally incompatible with classical finance since at the end of the evolutionary procedure the "rational"-pricing rule dominates the market and sets the pricing process.

Papers by Hens, Schenk-Hoppé \& Stalder ([14]) and Hens \& Schenk-Hoppé ([15]) opened the way for a completely new empirical framework in finance using this evolutionary set-up and performing simulations with real dividends data. Their simulations produced striking results, among them the fact that the Evolutionary Portfolio Rule is the only survivor even if in shorter time periods other rules succeed to some extent. Therefore, we were particularly motivated to extend and enhance their empirical framework, one direction being the exploration of the empirical results using a particular model for dividend formation.

Although many factor models explain the returns and prices of stocks, none exists for the dividends of stocks. However, stock payoffs are based on dividends, and, since these payoffs can be explained (even partially) by factor models, it is should be possible to predict some variation in dividends with a factor model of some short. In addition, we try to achieve many other goals by introducing a factor model for dividends. First, such a model offers an interesting approach to connecting dividends with macroeconomic variables. Moreover, factor models in multivariate analysis uncover a hidden structure within data, and this might be possible for dividends as well. This hidden structure might reveal aspects of dividends that cannot be discerned simply by observing the data. One reason for this is that managers artificially smooth the dividend process for framing purposes (Marsh \& Merton [20]). As a result, a hidden relationship might well be embedded in the empirical data and contribute substantially to the observed excess volatility phenomenon. More importantly, such a model could provide a richer ingredient in the evolutionary context and simulations.

With these points in mind, we offer a Dividend Factor Model that tries to expose some hidden volatility in the formation of dividends while connecting dividends with macroeconomic variables. The empirical calibration of this model is rather challenging and suggests new areas for research. More precisely, we have been confronted with two significant empirical problems. The first one was the non-stationary nature of the dividends data series. The other one was the severe correlations that exists between the macroeconomic variables. In order to deal with these empirical problems we have developed a modification of our Dividend Factor Model which led to a particular method of calibrating this model. By applying this method, we discovered surprising empirical results. More specifically, our method involves Principal Components Analysis (PCA) Regression techniques that have been used rarely in econometrical work. Our results indicate that there is a significant relation between the dividends of stocks and the transformed macroeconomic variables. In one case it was even plausible for an economic interpretation to be framed. These striking results support the use of PCA Regression method as an alternative solution to the widely known problem of multicollinearity in econometrics.

Finally, we performed a numerical analysis in order to study the long-run outcome of a competition given a new set of constant-proportions strategies in the aforementioned evolutionary context. The main incentive was to extend the present evolutionary empirical results and to propose new directions for research. Toward that end, we have included in our simulations the Evolutionary Portfolio Rule along with some new fixed-mix rules under artificial dividends generated from different models. More importantly, we have included the various calibrated versions of our particular Dividend Factor Model and we observed significant results. Our results should come as a useful addition to extant literature for both theoretical and practical reasons. In theoretical terms we reproduced an economy with real-life aspects, such as excess volatility, that support the notion of profitability for several fixed-mix rules in financial markets. In practical terms we have discovered some new fixed-mix strategies that could be profitable for investors at least in the short run. The last part of the simulations included dividends generated according to the calibrated estimates of our dividend model. We discovered that in our simulated economy there was a large amount of price volatility, although 
the Evolutionary Portfolio Rule was the clear winner of the contest. More significantly, in this particular "ecosystem" the volatility is not only very large but also very persistent over time.

This article is organized as follows. Section 2 provides a brief introduction to the model of Evolutionary Portfolio Theory. Important definitions and theorems are provided along with the general mathematical framework of the model. Section 3 presents our particular Dividend Factor Model and its particular method of calibration. Section 4 outlines the empirical methodology and the calibration procedure that was followed in relation to our particular dividend factor model. In Section 5 we present our numerical analysis along with some intuitive discussion about the significance of the results . Finally, Section 6 offers a comprehensive conclusion.

\section{The Evolutionary Portfolio Model}

In a series of papers, Hens, et. al. ([15, 14, 16]) and Evstigneev, et. al. ([8, 9]) formulate a theory for the Evolutionary Finance framework. They formulate their model as a stochastic dynamical system and provide necessary and sufficient conditions for the evolutionary stability of portfolio rules. According to these conditions, a stock market is evolutionary stable if and only if stocks are evaluated by the expected relative dividends. There are many versions and generalizations of this theory but we provide a brief summary of their simplest model which is sufficient for a brief introduction to this fascinating subject. For a detailed discussion and the full details of their model we refer the reader to their papers ([15, 14, 16],[8, 9]).

In this model the are $K$ long-lived assets with index $k=1,2, \ldots, K$ each paying an uncertain dividend $D_{t}^{k} \geq 0$ at any period in time which is indexed as $t=1,2 \ldots$ In this model the dividend denotes the total dividend of a company paid to all shareholders of asset $k$. In addition, this dividend depends on the possible states of nature that are determined in each period in time by the realization of a stationary stochastic process with values in some measurable space $(S, \mathcal{S})$. Moreover, $S$ is a finite set and every state $s$ is drawn with some strictly positive probability. Let $(\Omega, \mathcal{F}, \mathbb{P})$ be the probability space where $\Omega$ is the sample path space with representative element $\omega^{t}=\left(\ldots, \omega_{-1}, \omega_{0}, \omega_{1}, \ldots\right)$ where $\omega_{t} \in S$ is the state revealed at beginning of period $t$. Moreover, $\mathcal{F}=\mathcal{S}^{\mathbb{Z}}$ is the corresponding $\sigma$-algebra and $\mathbb{P}$ is the associated probability measure. The portfolio rule of investor $i$ is a fixed constant proportions strategy $\lambda^{i}=\left(\lambda_{0}^{i}, \lambda_{1}^{i}, \ldots, \lambda_{K}^{i}\right)$ with $\lambda_{k}^{i} \in[0,1]$ for all $k=1,2, \ldots, K$ and $\sum_{k=0}^{K} \lambda_{k}^{i}=1$. Here $\lambda_{k}^{i}$ is the fraction of wealth that investor $i$ assigns to the purchase of the asset $k$ and $\lambda_{0}^{i}$ is the fraction of wealth that each investors holds on cash. Investment strategies are distinct across investors for $i=1, \ldots, I$. Moreover, $\theta_{t, k}^{i}$ is the units of assets held by investor $i, w_{t}^{i}$ is the wealth of the investor $i$ and $p_{t}^{k}$ denotes the market clearing price of asset $k$ in period $t$. The price for cash is normalized so $p_{t}^{0}=1$. Therefore, the number of shares investor $i$ has multiplied by the price the stock equals the amount spent on this stock. This amount can be also expressed as the proportion of wealth spend on this stock. This is summarized in the following equation

$$
p_{t}^{k} \theta_{t, k}^{i}=\lambda_{k}^{i} w_{t}^{i}
$$

or

$$
\theta_{t, k}^{i}=\frac{\lambda_{k}^{i} w_{t}^{i}}{p_{t}^{k}}
$$

Thus $\theta_{t, k}^{i}$ is the number of the stock $k$ purchased by investor $i$ at time $t$ and can be expressed in terms of wealth, budget share and price as it appears in (2.1). If we sum the portfolios held by every investor, we will get the demand equation: $\sum_{i=1}^{I} \theta_{t, k}^{i} \equiv$ Demand. One important feature of this model is that demand equals supply so

$$
\sum_{i=1}^{I} \theta_{t, k}^{i}=s_{t}^{k}, \quad k=0,1, \ldots, K
$$

Supply is exogenous in this model and is normalized to one. Therefore,

$$
p_{t}^{k}=\sum_{i=1}^{I} \lambda_{k}^{i} w_{t}^{i}
$$


provides the equilibrium prices. The supply of cash is given by $\sum_{k=1}^{K} D_{t}^{k}\left(\omega^{t}\right)=s_{t}^{0}$. The budget constraint is given by

$$
w_{t}^{i}=\sum_{k=0}^{K} p_{t}^{k} \theta_{t, k}^{i}
$$

Equation (2.3) can be further written as

$$
w_{t+1}^{i}=\sum_{k=1}^{K}\left(D_{t+1}^{k}\left(\omega^{t+1}\right)+p_{t+1}^{k}\right) \theta_{t, k}^{i} .
$$

The amount of cash available in any period stems only from dividend payments and is consumed in every period. It is also assumed that all investors consume the same fraction of their wealth in all periods in time, i.e. $\lambda_{0}^{i}=\lambda_{0}$. This assumption ensures all strategies have the same savings rate and no strategy gains against others simply because it has a higher savings rate. Each agent consumes in each period $\lambda_{0} w_{t}^{i}$. Summing over all agents gives the total consumption expressed as $\sum_{i=1}^{I} \lambda_{0} w_{t}^{i}$. We define the total dividend of the economy as $D_{t} \equiv \sum_{k=1}^{K} D_{t}^{k}$ and the total wealth of the economy as $W_{t} \equiv \sum_{i=1}^{I} w_{t}^{i}$. We equate the total consumption of the economy to the total dividend in order to have

$$
W_{t}=\frac{D_{t}}{\lambda_{0}}
$$

The next step is to derive the market selection mechanism and for this we will need to start from (2.4) and divide both sides with $W_{t+1}$ and thus

$$
\frac{w_{t+1}^{i}}{W_{t+1}}=\sum_{k=1}^{K}\left[\left(\lambda_{0} \frac{D_{t+1}^{k}\left(\omega^{t+1}\right)}{D_{t+1}}+\frac{\sum_{i=1}^{I} \lambda_{k}^{i} w_{t+1}^{i}}{W_{t+1}}\right) \frac{\lambda_{k}^{i} w_{t}^{i}}{\sum_{i=1}^{I} \lambda_{k}^{i} w_{t}^{i}}\right] .
$$

If we define the investor's market shares (or relative returns) as $r_{t}^{i}=\frac{w_{t}^{i}}{W_{t}}$ and asset $k^{\prime} s$ relative dividend as $d_{t+1}^{k}={\frac{D_{t+1}^{k}}{D_{t+1}}}^{k}$ then we can rewrite $(2.5)$ as

$$
r_{t+1}^{i}=\sum_{k=1}^{K}\left[\left(\lambda_{0} d_{t+1}^{k}\left(\omega^{t+1}\right)+\sum_{i=1}^{I} \lambda_{k}^{i} r_{t+1}^{i}\right) \frac{\lambda_{k}^{i} r_{t}^{i}}{\sum_{i=1}^{I} \lambda_{k}^{i} r_{t}^{i}}\right] .
$$

Equation (2.6) is the market selection equation for the individual strategy and shows how wealth evolves through time for strategy $i$. In addition, we need a selection equation for all available strategies. We can define

$$
A_{t}^{i}=\lambda_{0} \sum_{k=1}^{K} d_{t+1} \frac{\lambda_{k}^{i} r_{t}^{i}}{\sum_{i=1}^{I} \lambda_{k}^{i} r_{t}^{i}} \quad \text { and } \quad B_{t, k}^{i}=\frac{\lambda_{k}^{i} r_{t}^{i}}{\sum_{i=1}^{I} \lambda_{k}^{i} r_{t}^{i}}
$$

so $(2.6)$ can be written as

$$
r_{t+1}^{i}=A_{t}^{i}+\sum_{k=1}^{K} B_{t, k}^{i} \boldsymbol{\lambda}_{\boldsymbol{k}} \mathbf{r}_{\mathbf{t}+\mathbf{1}}
$$

where $\boldsymbol{\lambda}_{\boldsymbol{k}}=\left(\lambda_{k}^{1}, \lambda_{k}^{2}, \ldots, \lambda_{k}^{I}\right)$ and $\mathbf{r}_{\mathbf{t}+\mathbf{1}}^{\mathbf{T}}=\left(r_{t+1}^{1}, r_{t+1}^{2}, \ldots, r_{t+1}^{I}\right)$. Thus we have in matrix notation

$$
\mathbf{r}_{\mathbf{t}+\mathbf{1}}=\mathbf{A}_{\mathbf{t}}+\mathbf{B}_{\mathbf{t}} \Lambda \mathbf{r}_{\mathbf{t}+\mathbf{1}}
$$

where $\boldsymbol{\Lambda}^{\boldsymbol{T}}=\left(\boldsymbol{\lambda}_{\mathbf{1}}^{\boldsymbol{T}}, \boldsymbol{\lambda}_{\mathbf{2}}^{\boldsymbol{T}}, \ldots, \boldsymbol{\lambda}_{\boldsymbol{K}}^{\boldsymbol{T}}\right) \in \mathbb{R}^{I \times K}, \mathbf{A}_{\mathbf{t}}^{\mathbf{T}}=\left(A_{t}^{1}, A_{t}^{2}, \ldots, A_{t}^{I}\right) \in \mathbb{R}^{I}$ and $\mathbf{B}_{\mathbf{t}}^{\mathbf{T}}=\left(B_{t}^{1}, \ldots, B_{t}^{I}\right) \in \mathbb{R}^{I}$. We can solve for $\mathbf{r}_{\mathbf{t}+\mathbf{1}}$ from (2.7) in order to get the market selection mechanism for all investors

$$
\mathbf{r}_{\mathbf{t}+\mathbf{1}}=\left[\mathbf{I}-\mathbf{B}_{\mathbf{t}} \boldsymbol{\Lambda}\right]^{-\mathbf{1}} \mathbf{A}_{\mathbf{t}}
$$

\footnotetext{
${ }^{1}$ In order this expression to be well defined we assume that at least one asset pays a dividend, $D_{t+1}>0$.
} 
where $\mathbf{I} \in \mathbb{R}^{I \times I}$ denotes the identity matrix. This equation governs the evolution of market shares for given trading strategies of investors and can be viewed as the market selection process in this evolutionary context. This evolution is well-defined if no bankruptcy occurs (i.e. $w_{t}^{i}>0$ ) and the market always clears (demand equals supply at all times).

Equation (2.8) can be also written as

$$
\mathbf{r}_{\mathbf{t}+\mathbf{1}}=\lambda_{0}\left[\mathbf{I}-\left[\frac{\lambda_{k}^{i} r_{t}^{i}}{\boldsymbol{\lambda}_{\boldsymbol{k}} \mathbf{r}_{\mathbf{t}}}\right]_{i}^{k} \boldsymbol{\Lambda}\right]^{-\mathbf{1}}\left[\sum_{k=1}^{K} d_{t+1}^{k}\left(\omega^{t+1}\right) \frac{\lambda_{k}^{i} r_{t}^{i}}{\boldsymbol{\lambda}_{\boldsymbol{k}} \mathbf{r}_{\mathbf{t}}}\right]_{i}
$$

and thus

$$
\mathbf{r}_{\mathbf{t}+\mathbf{1}}=h_{t}\left(\omega^{t+1}, \mathbf{r}_{\mathbf{t}}\right)
$$

where $h_{t}\left(\omega^{t+1}, \mathbf{r}_{\mathbf{t}}\right): \Delta^{I} \rightarrow \Delta^{I}$. Equation (2.10) generates a random dynamical system on the simplex

$$
\Delta^{I}=\left\{\mathbf{r} \in \mathbb{R}^{I} \mid r^{i} \geq 0, \sum_{i} r^{i}=1\right\} .
$$

We can define ${ }^{2}$

$$
\varphi(t, \omega, \mathbf{r}) \triangleq h\left(\omega^{t+1}, \cdot\right) \circ \ldots \circ h\left(\omega^{1}, \mathbf{r}\right)
$$

to be the vector of market shares at time $t$ for the initial distribution of market shares $\mathbf{r}$ and the sequence of realizations of states $\omega$. Then $\varphi: \mathbb{Z} \times \Omega \times \Delta^{I} \rightarrow \Delta^{I},(t, \omega, \mathbf{r}) \mapsto \varphi(t, \omega, \mathbf{r})$ is a $\mathcal{B}(\mathbb{Z}) \otimes \mathcal{F} \otimes \mathcal{B}\left(\Delta^{I}\right), \mathcal{B}\left(\Delta^{I}\right)$ measurable mapping for all $t \in \mathbb{Z}$ and $\omega \in \Omega$ where $\mathcal{B}$ denotes the Borel $\sigma$-algebra and $\mathcal{B}\left(\Delta^{I}\right) \triangleq \mathcal{B}\left(\mathbb{R}^{I} \cap \Delta^{I}\right)$. The following definition is particularly important since it describes fixed points:

Definition 2.1. A state $\overline{\mathbf{r}} \in \Delta^{I}$ is called a (deterministic) fixed point of $\varphi$ if, for all $\omega \in \Omega$ and all $t$,

$$
\overline{\mathbf{r}}=\varphi(t, \omega, \overline{\mathbf{r}}) .
$$

The vector of market shares $\overline{\mathbf{r}}$ is also said to be invariant under the market selection process (2.10).

By (2.11) and Definition 2.1 we see that a deterministic state is fixed under the one-state map if and only if it is under all $t$-step maps. The most obvious set of fixed points is the set of vertices of $\Delta^{I}$.

Although further analysis can be pursued in a more general setting for this model (see $[8,9])$ the following assumptions are imposed for the analysis that it will follow:

Assumption 2.2. I.i.d. dividend payments $d_{t}^{k}\left(\omega^{t}\right)=d^{k}\left(\omega_{t}\right)$, for all $k=1,2, \ldots K$ and the state of nature $\omega_{t}$ follows an i.i.d. process.

Assumption 2.3. Absence of redundant assets, i.e. the matrix of relative dividend payments $\left(d^{k}(s)\right)_{s \in S}^{k=1, \ldots, K}$ has full rank.

The following Proposition is of particular importance since it relates fixed points with unique portfolio rules.

Proposition 2.4. All portfolio rules with positive wealth are identical at a deterministic fixed point.

Proof. See [9].

Proposition 2.4 states that $\lambda^{j}$ and $\lambda^{i}$ are identical at a fixed point so fixed points are associated with unique portfolio rules. In addition, we need the following stability definitions of the fixed points. The first definition describes stability with respect to every new portfolio rule and is given below:

Definition 2.5. A portfolio rule $\lambda^{i}$ is called evolutionary stable, if for every portfolio rule $\lambda^{j} \neq \lambda^{i}$ there is a random variable $\varepsilon>0$ such that $\lim _{t \rightarrow \infty} \varphi^{i}(t, \omega, \mathbf{r})=1$ for all $r^{i} \geq 1-\varepsilon(\omega)$ almost surely. ${ }^{3}$

The second definition describes stability with respect to local innovations which are portfolio rules that are restricted to small deviations from the dominant rule.

Definition 2.6. A portfolio rule $\lambda^{i}$ is called locally evolutionary stable, if there exists a random variable $\delta(\omega)>0$ such that $\lambda^{i}$ is evolutionary stable for all portfolio rules $\lambda^{j} \neq \lambda^{i}$ with $\left\|\lambda^{i}(\omega)-\lambda^{j}(\omega)\right\|<\delta(\omega)^{4}$

\footnotetext{
${ }^{2}$ Where we have used $\triangleq$ as the notation for defining an expression.

${ }^{3}$ Where $\varphi^{i}$ is the function as defined in (2.11) for the strategy $i$.

${ }^{4}$ Where $\|\cdot\|$ denotes the Euclidean distance in the Simplex $\Delta^{I}$.
} 
for all $\omega$.

Stability implies that small perturbations do not have any long run impact in the equilibrium process. In the case of Evolutionary Portfolio Theory, a portfolio rule that is stable cannot be ruled away from an innovation, i.e. a new portfolio rule. Therefore, an equilibrium in the economy can be expressed mathematically as a fixed point in a dynamical system. In this fixed point, there exists only one portfolio rule that has conquered all the market wealth and sets the prices. The fact that some investors can decide to play a different portfolio rule, viewed as innovation in our context, can be expressed mathematically as a perturbation of the dynamical system. The fixed point where all the possible innovations can be driven out of the market can be considered stable and its unique portfolio rule is the best available strategy. ${ }^{5}$

The main result is that these stability conditions lead to a unique evolutionary portfolio rule that is stable assuming the relative dividend payoffs are governed by an i.i.d process. ${ }^{6}$ In order to analyze evolutionary stability we should consider the market selection mechanism given by (2.9) with two portfolio rules. One rule should be considered as the incumbent (with market share denoted as $r_{t}^{1}$ ) and the other as the mutant (market share denoted by $r_{t}^{2}$ which is equal in this case with $1-r_{t}^{1}$ ). Thus

$$
\left[\begin{array}{c}
r_{t+1}^{1} \\
r_{t+1}^{2}
\end{array}\right]=\frac{\lambda_{0}}{\Delta_{t+1}}\left[\begin{array}{cc}
1-\sum_{k=1}^{K} \lambda_{k}^{2} \theta_{t, k}^{2} & \sum_{k=1}^{K} \lambda_{k}^{2} \theta_{t, k}^{1} \\
\sum_{k=1}^{K} \lambda_{k}^{1} \theta_{t, k}^{2} & 1-\sum_{k=1}^{K} \lambda_{k}^{1} \theta_{t, k}^{1}
\end{array}\right] \cdot\left[\begin{array}{l}
\sum_{k=1}^{K} d_{t+1}^{k}\left(\omega_{t+1}\right) \theta_{t, k}^{1} \\
\sum_{k=1}^{K} d_{t+1}^{k}\left(\omega_{t+1}\right) \theta_{t, k}^{2}
\end{array}\right]
$$

where $\Delta_{t+1}$ is the following determinant

$$
\Delta_{t+1}=\left[1-\sum_{k=1}^{K} \lambda_{k}^{1} \theta_{t, k}^{1}\right]\left[1-\sum_{k=1}^{K} \lambda_{k}^{2} \theta_{t, k}^{2}\right]-\left[\sum_{k=1}^{K} \lambda_{k}^{2} \theta_{t, k}^{1}\right]\left[\sum_{k=1}^{K} \lambda_{k}^{1} \theta_{t, k}^{2}\right] .
$$

So the return for the incumbent strategy is

$$
r_{t+1}^{1}=\frac{\lambda_{0}}{\Delta_{t+1}}\left[1-\sum_{k=1}^{K} \lambda_{k}^{2} \theta_{t, k}^{2}\right]\left[\sum_{k=1}^{K} d_{t+1}^{k}\left(\omega_{t+1}\right) \theta_{t, k}^{1}\right]+\left[\sum_{k=1}^{K} \lambda_{k}^{2} \theta_{t, k}^{1}\right]\left[\sum_{k=1}^{K} d_{t+1}^{k}\left(\omega_{t+1}\right) \theta_{t, k}^{2}\right] .
$$

In dynamical systems we consider the linearization of the local dynamics at the fixed points, where in our case $r_{t}^{1}=1$. Therefore, we need the following derivative

$$
\begin{aligned}
\left.\frac{\partial h\left(\omega^{t+1}, r_{t}^{1}\right)}{\partial r_{t}^{1}}\right|_{r_{t}^{1}=1}= & \\
= & \frac{\lambda_{0}}{\left(\lambda_{0}\right)^{2}}\left[\lambda_{0}\left(\sum_{k=1}^{K} d_{t+1}^{k}\left(\omega_{t+1}\right) \frac{\lambda_{k}^{2}}{\lambda_{k}^{1}}-\sum_{k=1}^{K} \lambda_{k}^{2} \frac{\lambda_{k}^{2}}{\lambda_{k}^{1}}-\left(1-\lambda_{0}\right) \sum_{k=1}^{K} d_{t+1}^{k}\left(\omega_{t+1}\right) \frac{\lambda_{k}^{2}}{\lambda_{k}^{1}}\right)-\right. \\
& \left.-\left(\sum_{k=1}^{K} d_{t+1}^{k}\left(\omega_{t+1}\right)\right)\left(\left(1-\lambda_{0}\right) \sum_{k=1}^{K} \lambda_{k}^{1} \frac{\lambda_{k}^{2}}{\lambda_{k}^{1}}-\lambda_{0} \sum_{k=1}^{K} \lambda_{k}^{2} \frac{\lambda_{k}^{2}}{\lambda_{k}^{1}}-\sum_{k=1}^{K} \lambda_{k}^{1} \frac{\lambda_{k}^{2}}{\lambda_{k}^{1}}\right)\right] \\
= & \sum_{k=1}^{K}\left[\left(\lambda_{0} d_{t+1}^{k}\left(\omega_{t+1}\right)+\lambda_{k}^{1}\right) \frac{\lambda_{k}^{2}}{\lambda_{k}^{1}}\right]
\end{aligned}
$$

where we use $\left.\frac{\partial \theta_{t, k}^{1}}{\partial r_{t}^{1}}\right|_{r_{t}^{1}=1}=\frac{\lambda_{k}^{2}}{\lambda_{k}^{1}}$ and $\operatorname{thus}^{7}$

$$
\left.\frac{\partial h\left(\omega^{t+1}, r_{t}^{1}\right)}{\partial r_{t}^{1}}\right|_{r_{t}^{1}=1}=1-\lambda_{0}+\lambda_{0} \sum_{k=1}^{K} d_{t+1}^{k}\left(\omega_{t+1}\right) \frac{\lambda_{k}^{2}}{\lambda_{k}^{1}}
$$

\footnotetext{
${ }^{5}$ Best response in the evolutionary game theoretical framework (i.e. ESS).

${ }^{6}$ In the more general version of this model this assumption is weaker. It requires stationary and ergodic dividend payoffs.

${ }^{7}$ Equation (2.12) is useful in the general model where strategies are not simple and therefore capital gains exist (expressed as $\left.\lambda_{k}^{1}\left(\omega^{t+1}\right) \frac{\lambda_{k}^{2}\left(\omega^{t}\right)}{\lambda_{k}^{1}\left(\omega^{t}\right)}\right)$. See Evstigneev, et. al. [9] for more details on this issue.
} 
In dynamical systems one can look at the Lyapunov exponent in order to see whether a system is stable or not. If the exponent is negative the system is stable and if the exponent is positive the system is unstable. Using (2.13) and the fact that the system is random, we get the following Lyapunov exponent

$$
g_{\lambda^{1}}\left(\lambda^{2}\right)=\int_{S} \ln \left[1-\lambda_{0}+\lambda_{0} \sum_{k=1}^{K} d_{t+1}^{k}(s) \frac{\lambda_{k}^{2}}{\lambda_{k}^{1}}\right] d \mathbb{P}
$$

where integration takes place with respect to the associated probability measure $\mathbb{P}$. Equation (2.14) can be written more generally for two strategies $i$ and $j$ as

$$
g_{\lambda^{j}}\left(\lambda^{i}\right)=\mathbb{E}\left[\ln \left(1-\lambda_{0}+\lambda_{0} \sum_{k=1}^{K} d_{t+1}^{k}(s) \frac{\lambda_{k}^{i}}{\lambda_{k}^{j}}\right)\right]
$$

where (2.15) can be interpreted as the exponential growth rate ${ }^{8}$ of portfolio rule $\lambda^{i}$ at $\lambda^{j}$-prices. If the growth rate is negative, $g_{\lambda^{j}}\left(\lambda^{i}\right)<0$, portfolio rule $\lambda^{i}$ loses market share while $\lambda^{j}$ 's market share tends to one. Therefore, portfolio rule $\lambda^{j}$ is considered stable against $\lambda^{i}$. On the contrary, if the growth rate is positive $g_{\lambda^{j}}\left(\lambda^{i}\right)>0$, portfolio rule $\lambda^{i}$ gains market share while $\lambda^{j}$ 's market share falls. Thus, portfolio rule $\lambda^{j}$ is considered unstable against $\lambda^{i}$. Evstigneev, Hens, and Schenk-Hoppé ([9]) show the following significant result:

Theorem 2.7. The portfolio rule $\lambda^{*}$, defined by

$$
\lambda_{k}^{*}=\left(1-\lambda_{0}\right) \mathbb{E} d^{k}(s), \quad k=1,2, \ldots, K
$$

is the only investment strategy that is evolutionary stable against any other portfolio rule. That is, $g_{\lambda^{*}}(\lambda)<0$ and $g_{\lambda}\left(\lambda^{*}\right)>0$ for all $\lambda \neq \lambda^{*}$.

Proof. See ([9]) or ([21]).

Equation (2.15) shows that under-diversification is fatal for investment. This leads to the following corollary:

Corollary 2.8. Suppose some incumbent rule $\lambda^{j}$ with $\lambda_{k}^{j}=0$ for some asset $\mathrm{k}$ has conquered the market. Then any portfolio rule $\lambda^{i}$ with $\lambda_{k}^{i}>0$ for all $\mathrm{k}$ grows against $\lambda^{j}$, i.e. $g_{\lambda^{j}}\left(\lambda^{i}\right)>0$. In fact the growth rate is arbitrarily large.

The following remark shows the relation between the evolutionary portfolio rule and the "betting your beliefs" strategy.

Remark 2.9. The evolutionary portfolio rule $\lambda_{k}^{*}=\left(1-\lambda_{0}\right) \mathbb{E} d^{k}(s)$ reduces to the "betting your beliefs" strategy, i.e. $\lambda_{s}=p_{s}$ when assets are arrow securities.

Remark 2.9 is true since arrow securities pay one in only one state and zero otherwise.

As a final note, we would like to point out the most important implication of this model. The stability criterion of the evolutionary rule may help to theoretically describe and explain empirically the fact that, in the long term, stock markets look rational while, in the short term, economic profit is available from excess volatility.

\section{A Factor Model for Dividends}

\subsection{Motivation for the Dividend Factor Model}

Although there are many factor models for returns and prices of stocks there are not so many, if there are any at all, for the dividends of stocks. One should not just imitate models that are currently available to stocks for dividends since the latter have their own peculiarities and in many aspects are different from stocks. However, stock payoffs are based on dividends and since these payoffs can be explained (even partially) from

\footnotetext{
${ }^{8}$ For an intuitive analysis on exponential growth rates in this context see Luenberger ch. 15 ([19])
} 
factor models, it is quite possible to explain some variation on dividends with a factor model. As in the stock case, where there are many and different factor models that try to shed light in price variation, there might be many different factor-like models that can shed some light on the dividends. Thus, our particular model can been seen as only one attempt and by no means should be seen as the only valid one that can explain everything for dividend formation. We try to achieve many goals simultaneously by introducing a factor-like model for dividends. First of all, a model like that is on its own a very interesting approach for connecting dividends with macro-economic factors. Moreover, in multivariate analysis, factor models are used to uncover a hidden structure that lies on the data and that might also be possible for dividends as well. This hidden structure might reveal aspects on dividends that are absent from simply observing the data. This is possible because managers tend to artificially smooth the dividend process for framing purposes. ${ }^{9}$ Toward that end, a hidden relationship could be embedded in empirical data that contributes substantially to the observed excess volatility phenomenon. Finally, and more importantly, a model like that could contribute a different aspect and provide a richer ingredient in the evolutionary context and simulations.

As noted above, a hybrid model is needed for dividends rather than a simple factor model copied from stocks. This is true because dividends have their own characteristics. For example, although dividends could be modeled in continuous time, their aspects are best captured in a discrete time framework. In addition, dividends cannot be negative like returns. To that end, lognormal distribution can be seen as the appropriate starting model for dividends. This precludes dividends from being zero but nevertheless this is a welcome aspect since stocks that pay no dividends may introduce a bias on the Evolutionary Finance model. Geske ([13]) provides some explanation why lognormal dividends should be a reasonable assumption for dividends. Dividends are also different with respect to stocks in terms of the idiosyncratic components. Contrary to stocks, there is no theory that is well-known for portfolio of dividends and hence dividends can depend on some factors that they do not have in common. ${ }^{10}$ Taking all these considerations in mind, we propose a model for the total dividend of a company that follows a lognormal process.

\subsection{The Dividend Factor Model}

There are $i=1, \ldots K$ companies in the economy that pay a total dividend denoted by $D_{i}^{t}$ where $t$ is the time index and $i$ denotes a particular company. Time is discrete and measured usually in years. One time period is a year. Dividends are distributed at the end of the year and they follow a stationary process.

There are also $j=1 \ldots m$ factors denoted by $X_{j}$ and are jointly normally distributed with some mean and variance and thus

$$
\mathbf{X} \sim \mathbf{N}_{\mathbf{m}}\left(\mu_{\mathbf{X}}, \Sigma_{\mathbf{X}}\right)
$$

where

$$
\mathbf{X}=\left(\begin{array}{c}
X_{1} \\
\vdots \\
X_{m}
\end{array}\right), \quad \boldsymbol{\mu}_{\mathbf{X}}=\left(\begin{array}{c}
\mu_{X_{1}} \\
\vdots \\
\mu_{X_{m}}
\end{array}\right)
$$

and

$$
\boldsymbol{\Sigma}_{\boldsymbol{x}}=\left(\begin{array}{cccc}
\operatorname{var}\left(X_{1}\right) & \operatorname{cov}\left(X_{1}, X_{2}\right) & \ldots & \operatorname{cov}\left(X_{1}, X_{m}\right) \\
\operatorname{cov}\left(X_{2}, X_{1}\right) & \operatorname{var}\left(X_{2}\right) & & \\
\vdots & & \ddots & \\
\operatorname{cov}\left(X_{m}, X_{1}\right) & \ldots & & \operatorname{var}\left(X_{m}\right)
\end{array}\right)
$$

In addition, the above variance-covariance matrix is assumed to have distinct and positive eigenvalues i.e.

$$
\lambda_{1}>\lambda_{2}>\ldots>\lambda_{m}>0
$$

Moreover, every element in $\mathbf{X}$ is considered a white noise process in time. ${ }^{11}$ Let $\xi_{i j}$ denote the sensitivity of the total dividend of a company $i$ with a factor $X_{j}$, that remains constant through time, then the total dividend of a company is given by,

\footnotetext{
${ }^{9}$ The so called Mash-Merton critic ([20]) on Shiller's work.

${ }^{10}$ This is an alternative way to incorporate idiosyncratic components to the model that relates well to our evolutionary simulation framework.

${ }^{11}$ Where we define as white noise an i.i.d process with finite mean and variance.
} 


$$
D_{i}^{t}=\exp \left(\sum_{j=1}^{m} \xi_{i j} X_{j}^{t}\right)
$$

It is immediate from the assumptions for the model above and the well-known property from linear algebra which is given below that (3.2) is a positive-definite matrix.

Property 3.1.

$$
\mathbf{x}^{\mathbf{T}} \mathbf{\Sigma} \mathbf{x} \text { is positive definite } \Leftrightarrow \text { all eigenvalues of } \mathbf{\Sigma} \text { are positive. }
$$

In addition, the following well-known property from the theory of the normal multivariate distribution is particularly useful:

Property 3.2. If $\mathbf{X}$ is jointly normally distributed, denoted by $\mathbf{X} \sim \mathbf{N}_{\mathbf{p}}(\boldsymbol{\mu}, \boldsymbol{\Sigma})$, then any linear combination of variables $\mathbf{a}^{\mathbf{T}} \mathbf{X}$ is distributed as $N\left(\mathbf{a}^{\mathbf{T}} \boldsymbol{\mu}, \mathbf{a}^{\mathbf{T}} \mathbf{\Sigma} \mathbf{a}\right)$. In addition, if $\mathbf{a}^{\mathbf{T}} \mathbf{X}$ is distributed as $N\left(\mathbf{a}^{\mathbf{T}} \boldsymbol{\mu}, \mathbf{a}^{\mathbf{T}} \mathbf{\Sigma} \mathbf{a}\right)$ for every $\mathbf{a}$, then $\mathbf{X} \sim \mathbf{N}_{\mathbf{p}}(\boldsymbol{\mu}, \boldsymbol{\Sigma})$.

Proof. See proof in Anderson ([1]).

Furthermore, 3.2 lead us to the following property:

Property 3.3. The marginal distribution of any component $X_{j}$ of $\mathbf{X}$ is normally distributed i.e.

$$
X_{j} \sim\left(\mu_{X_{j}}, \sigma_{X_{j}}^{2}\right)
$$

where $\sigma_{X_{j}}^{2}$ denotes the variance of the factor $X_{j}$.

Proof. This is a direct consequence of property 3.2 and the initial assumptions of this model.

Equation (3.3) is equivalent to

$$
\ln D_{i}^{t}=\sum_{j=1}^{m} \xi_{i j} X_{j}^{t} .
$$

Immediately, it can be seen from the property above that equation (3.4) follows a normal distribution since it is the linear combination of normal variables and thus equation (3.3) follows a lognormal distribution since it is the exponent of a normal variable. Hence, dividends are assumed to follow a lognormal process. The lognormal assumption does not handle companies that pay no dividends at all. However, since the mainstream model in price valuation is the expected discounted dividends model, companies that pay nothing and have other methods of their stock price valuation should not take part in any comparison simulation that uses as a basis for price formation the expected discounted dividends model. In addition, since the lhs of (3.3) is the total dividend, a small amount in one year could be interpreted as a zero dividend for that particular year. Therefore, the effect of a bad year could be accommodated also with (3.3) practically speaking.

Then we will have the following system of equations

$$
\begin{array}{rlr}
\ln D_{1}^{t} & =\xi_{11} X_{1}^{t}+\ldots+\xi_{1 m} X_{m}^{t} \\
\ln D_{2}^{t} & =\xi_{21} X_{1}^{t}+\ldots+\xi_{2 m} X_{m}^{t} \\
\vdots & = & \vdots \\
\ln D_{K}^{t} & =\xi_{K 1} X_{1}^{t}+\ldots+\xi_{K m} X_{m}^{t} .
\end{array}
$$

This can be written in matrix form as

$$
\mathbf{D}=\mathbf{\Xi} \cdot \mathbf{X}
$$

where

$$
\mathbf{D}=\left(\begin{array}{c}
\ln D_{1}^{t} \\
\vdots \\
\ln D_{K}^{t}
\end{array}\right), \mathbf{X}=\left(\begin{array}{c}
X_{1}^{t} \\
\vdots \\
X_{m}^{t}
\end{array}\right), \boldsymbol{\Xi}=\left(\begin{array}{ccc}
\xi_{11} & \ldots & \xi_{1 m} \\
\vdots & \ddots & \vdots \\
\xi_{m 1} & \ldots & \xi_{K m}
\end{array}\right)
$$


or

$$
\mathbf{D}_{\mathbf{T}}=\boldsymbol{\Xi} \cdot \mathbf{X}_{\mathbf{T}}
$$

with

$$
\mathbf{D}_{\mathbf{T}}=\left(\begin{array}{ccc}
\ln D_{1}^{1} & \ldots & \ln D_{1}^{T} \\
\vdots & \ddots & \vdots \\
\ln D_{K}^{1} & \ldots & \ln D_{K}^{T}
\end{array}\right), \mathbf{X}_{\mathbf{T}}=\left(\begin{array}{ccc}
X_{1}^{1} & \ldots & X_{1}^{T} \\
\vdots & \ddots & \vdots \\
X_{m}^{1} & \ldots & X_{m}^{T}
\end{array}\right), \boldsymbol{\Xi}=\left(\begin{array}{ccc}
\xi_{11} & \ldots & \xi_{1 m} \\
\vdots & \ddots & \vdots \\
\xi_{K 1} & \ldots & \xi_{K m}
\end{array}\right)
$$

We can use the assumption given by (3.1) for the following result:

Result 3.4. Given our assumption in (3.1) the vector $\mathbf{D}$ is jointly normally distributed i.e.

$$
\mathbf{D} \sim \mathbf{N}_{\mathbf{K}}\left(\boldsymbol{\Xi} \mu_{X}, \boldsymbol{\Xi} \boldsymbol{\Sigma}_{X} \boldsymbol{\Xi}^{\mathbf{T}}\right)
$$

where $\boldsymbol{\Sigma}_{\boldsymbol{X}}$ is defined as in (3.2). Moreover,

$$
\ln D_{i}^{t} \sim N\left(\boldsymbol{\xi}_{i}^{\mathbf{T}} \boldsymbol{\mu}, \boldsymbol{\xi}_{\boldsymbol{i}}^{\mathbf{T}} \boldsymbol{\Sigma}_{\boldsymbol{X}} \boldsymbol{\xi}_{\boldsymbol{i}}\right) \quad \text { for every } i=1,2, \ldots, K
$$

where $\boldsymbol{\xi}_{\boldsymbol{i}}^{\mathbf{T}}=\left(\xi_{i 1}, \xi_{i 2}, \ldots, \xi_{i m}\right)$.

Proof. This result follows as a direct application of properties 3.2 and 3.3.

Of equal importance is the assumption that every element in $\mathbf{X}$ is considered a white noise process in time and the following results are direct consequences of that assumption.

Result 3.5. The random variables of the process $\left\{\ln D_{i}^{t}\right\}_{t=1, \ldots, T}$ for every $i=1,2, \ldots, K$ are $i . i . d$ (independently and identically distributed) in time.

Proof. A linear combination of normal variables is normal and thus $\ln D_{i}$ is normal. Since $X_{j}$ is i.i.d. for every $j=1, \ldots, m$ then $\ln D_{i}^{t} \sim N\left(\boldsymbol{\xi}_{\boldsymbol{i}}^{\mathbf{T}} \boldsymbol{\mu}, \boldsymbol{\xi}_{\boldsymbol{i}}^{\mathbf{T}} \boldsymbol{\Sigma}_{\boldsymbol{X}} \boldsymbol{\xi}_{\boldsymbol{i}}\right)$ for every $t=1, \ldots, T$.

The main point here is that since the log of the dividends for each company are i.i.d the evolutionary theorems hold. The next result is especially useful in the empirical econometric work.

Result 3.6. The process $\left\{\ln D_{i}^{t}\right\}_{t=1, \ldots,, T}$ for every $i=1,2, \ldots, K$ is weakly stationary.

Proof. See ([21]) on page 32 .

Remark 3.7. For a multivariate Gaussian process weak stationarity implies strict stationarity.

This is a direct consequence of the fact that Gaussian processes are fully characterized by their first two moments.

In our particular case we also have:

Result 3.8. The process through time $\left\{\ln D_{i}^{t}\right\}$ for every $i=1,2, \ldots, K$ is ergodic.

Proof. This is a direct consequence of the result 3.5 and the fact that the ergodic theorem ${ }^{12}$ is a generalization of the strong law of large numbers.

In practice, it is very difficult to encounter an i.i.d time series, so the assumption that $\left\{\ln D_{i}^{t}\right\}_{t}$ is i.i.d. can be relaxed. We require only that $\left\{\ln D_{i}^{t}\right\}_{t}$ is stationary and ergodic.

In our model we have not included any idiosyncratic components since there is no theory that can deal with them later on. According to economic theory, for the pricing of stocks, idiosyncratic risks should not matter because we can form sufficiently diversified portfolios. However, although some dividends can have common factors that influence them, it can also have some idiosyncratic components. We model these components with the inclusion of additional factors where necessary, allowing $\xi_{i j}$ 's to take specific values in order to include that effect whenever necessary.

This model introduces some hidden volatility in the formation of dividends since the log of the dividends change every year as the variation changes through the factors of the economy. Someone can also center the mean of the factors around zero thus try to measure the change in dividends with respect to unanticipated change in factors. Although, this might be true for the stocks, it is not clear if it is also true for the dividends of the stocks.

\footnotetext{
${ }^{12}$ For the proof of the theorem and further information on ergodicity see ([3]).
} 


\subsection{The Dividend Factor Model using PCA}

Direct econometric estimation of the general dividends factor model is empirically not efficient due to multicollinearity issues. The following result summarizes the application of PCA in the general dividends factor model.

Result 3.9. Let $\mathbf{x}$ be a vector of $n$ random variables, $x_{i}$, with mean vector $\boldsymbol{\mu}$ and variance-covariance matrix $\mathbf{\Sigma}_{\mathbf{x}}$. Define $\mathbf{X}=\mathbf{x}-\boldsymbol{\mu}$ and set $\mathbf{z}=\mathbf{E}^{\mathbf{T}} \mathbf{X}$ where the $\mathrm{k}$ th column in matrix $\mathbf{E}$ corresponds to the $\mathrm{k}$ th eigenvector $\mathbf{e}_{\mathbf{k}}$ of the variance-covariance matrix $\mathbf{\Sigma}_{\boldsymbol{X}}$. Now, each element of $\mathbf{z}, z_{i}$ is called the mean-adjusted principal component of $x_{i}$. Then, $\mathbf{x} \sim \mathbf{N}_{\mathbf{n}}\left(\boldsymbol{\mu}, \mathbf{\Sigma}_{\mathbf{x}}\right)$ implies that

$$
\mathbf{z} \sim \mathbf{N}_{\mathbf{n}}(\mathbf{0}, \boldsymbol{\Lambda})
$$

where $\boldsymbol{\Lambda}$ is a diagonal eigenvalue matrix. Moreover, in case that $\mathbf{E}^{\mathbf{T}}$ includes only the first $m$ columns of $\mathbf{E}$ then

$$
\mathbf{z} \sim \mathbf{N}_{\mathbf{m}}\left(\mathbf{0}, \Lambda_{\mathbf{m}}\right)
$$

where $\boldsymbol{\Lambda}_{\mathbf{m}}$ is the $(m \times m)$ partition of $\boldsymbol{\Lambda}$.

Actually, it is clear from the result above that any arbitrary subset of $\mathbf{z}$ is jointly normally distributed. What we need to do is to rearrange elements of $\mathbf{z}$ and their corresponding mean and variances in order to apply the result above. The following result is used directly in the simulations of the PCA Dividend Factor Model.

Result 3.10. The mean-adjusted principal component is normally distributed i.e. $z_{i} \sim N\left(0, \lambda_{i}\right)$ for every $i=1, \ldots, m$ and $z_{i}$ is independent of $z_{j}$ for every $i \neq j$.

It is usually convenient in practice to apply PCA directly in mean-adjusted variables. Our model given by (3.3) must be modified slightly in order to allow the correct econometric estimation. Thus in the $P C A$ Dividend Factor Model we substitute (3.3) with

$$
D_{i}^{t}=\exp \left(a_{i}+\sum_{j=1}^{m} \xi_{i j} z_{j}^{t}\right)
$$

where $a_{i}$ is the corresponding constant of regression.

\subsection{Application of PC Regression in the Dividend Factor Model}

For the application of the PCA in our model, we need to start with a variance-covariance matrix of appropriate variables that is possible to influence dividends. We will provide below the steps that we have followed in order to calibrate the $\boldsymbol{\Xi}$ matrix.

Step 1.

Form the following $(T \times m)$ matrix

$$
\mathbf{x}=\left[\begin{array}{cccc}
x_{1}^{1} & x_{2}^{1} & \ldots & x_{m}^{1} \\
x_{1}^{2} & x_{2}^{2} & \ldots & x_{m}^{2} \\
\vdots & \vdots & \ddots & \vdots \\
x_{1}^{T} & x_{2}^{T} & \ldots & x_{m}^{T}
\end{array}\right]
$$

where each column has a variable with $T$ observations that might influence the dividends of a company.

Step 2.

Subtract the mean for every variable $k=1, \ldots, m$ so

$$
X_{k}^{t}=x_{k}^{t}-\bar{x}_{k}=x_{k}^{t}-\frac{1}{T} \sum_{t=1}^{T} x_{k}^{t}
$$


and so form the following matrix

$$
\mathbf{X}=\left[\begin{array}{ccc}
X_{1}^{1} & \ldots & X_{m}^{1} \\
\vdots & \ddots & \vdots \\
X_{1}^{T} & \ldots & X_{m}^{T}
\end{array}\right]
$$

Step 3.

Use the $\mathbf{X}$ from above and calculate the variance-covariance matrix

$$
\boldsymbol{\Sigma}_{\boldsymbol{X}}=\frac{1}{T-1} \mathbf{X}^{\mathbf{T}} \mathbf{X}
$$

where $\sigma_{i j}=\operatorname{cov}\left(x_{i}, x_{j}\right)$ is the element of the matrix above and denotes the covariance between one of the factors, $x_{i}$, that influences dividends with another factor, $x_{j}$, that influences dividends as well.

Step 4.

For the matrix $\boldsymbol{\Sigma}_{\boldsymbol{X}}$ in step 3 calculate the eigenvalues and eigenvectors and put them in order as pairs

$$
\lambda_{1}>\lambda_{2}>\ldots>\lambda_{m}, \quad \mathbf{e}_{\mathbf{1}}, \mathbf{e}_{\mathbf{2}}, \ldots, \mathbf{e}_{\mathbf{m}}
$$

so

$$
\left(\lambda_{1}, \mathbf{e}_{\mathbf{1}}\right) \quad \ldots \quad\left(\lambda_{m}, \mathbf{e}_{\mathbf{m}}\right)
$$

$\underline{\text { Step } 5 .}$

Form the eigenmatrix $\mathbf{E}$ as

$$
\mathbf{E}=\left[\begin{array}{lll}
\mathbf{e}_{1} & \ldots & \mathbf{e}_{\mathbf{m}}
\end{array}\right]
$$

where $\mathbf{e}_{\mathbf{j}}$ denotes the corresponding eigenvector.

Step 6.

Calculate the PC as the system

$$
\mathbf{Z}=\mathbf{X E}
$$

where $\mathbf{Z}$ and $\mathbf{E}$ are defined in step 2 and step 5 above. Thus,

$$
z_{k}^{t}=\sum_{j=1}^{m} e_{k j} X_{j}^{t}
$$

and

$$
\operatorname{var}\left(z_{k}\right)=\lambda_{k} .
$$

Step 7.

Estimate the following regression equation for $i=1, \ldots, K$, the total number of stocks considered in the model

$$
\mathbf{D}_{\mathrm{i}}=\tilde{\mathbf{Z}} \gamma_{i}+\varepsilon
$$

where

$$
\mathbf{D}_{\mathbf{i}}=\left[\begin{array}{c}
\ln D_{i}^{1} \\
\vdots \\
\ln D_{i}^{T}
\end{array}\right], \quad \gamma_{\boldsymbol{i}}=\left[\begin{array}{c}
a_{i} \\
\gamma_{i 1} \\
\vdots \\
\gamma_{i m}
\end{array}\right]
$$

and

$$
\tilde{\mathbf{Z}}=\left[\begin{array}{cccc}
1 & z_{1}^{1} & \ldots & z_{m}^{1} \\
1 & z_{1}^{2} & \ldots & z_{m}^{2} \\
\vdots & \vdots & \ddots & \vdots \\
1 & z_{1}^{T} & \ldots & z_{m}^{T}
\end{array}\right]
$$

Estimate the vector $\gamma_{i}$ as

$$
\hat{\gamma}_{i}=\left(\tilde{\mathbf{Z}}^{\mathrm{T}} \tilde{\mathbf{Z}}\right)^{-1} \tilde{\mathbf{Z}}^{\mathrm{T}} \mathbf{D}_{\mathbf{i}}
$$


Therefore, form matrix $\boldsymbol{\Xi}$ as

$$
\Xi=\Gamma
$$

where

$$
\boldsymbol{\Gamma}=\left[\begin{array}{cccc}
\hat{\gamma}_{11} & \hat{\gamma}_{12} & \ldots & \hat{\gamma}_{1 m} \\
\hat{\gamma}_{21} & \hat{\gamma}_{22} & \ldots & \hat{\gamma}_{2 m} \\
\vdots & \vdots & \ddots & \vdots \\
\hat{\gamma}_{K 1} & \hat{\gamma}_{K 2} & \ldots & \hat{\gamma}_{K m}
\end{array}\right]
$$

Step 9.

If necessary, reduce dimensionality and calculate the regression equation

$$
\mathbf{D}_{\mathbf{i}}=\tilde{\mathbf{Z}}_{\mathbf{m}^{\prime}} \gamma_{m^{\prime} i}+\varepsilon_{m^{\prime}}
$$

for every stock $i=1, \ldots, K$ where now $\mathbf{m}^{\prime}<\mathbf{m}$. A common practice that is used to determine the number of principal components that should be included in the regression is to examine the scree plot, which is a plot of the eigenvalue $\lambda_{i}$ against $i$, and to decide at which point there is an elbow in the graph. ${ }^{13}$ Another common practice is to look at a criterion such as the cumulative proportion of the total variance. For a detailed discussion on selection criteria for choosing the appropriate number of principal components we point the reader to see Ch. 6 in Jolliffe ([18]).

In this case,

$$
\Xi=\Gamma_{m^{\prime}}
$$

where $\boldsymbol{\Gamma}_{\boldsymbol{m}^{\prime}}$ is the reduced $\left(k \times m^{\prime}\right)$ matrix where $m^{\prime}<m$ since we have included $m^{\prime}$ principal components in our regression equation.

We can follow the same steps above when we use the correlation matrix $\boldsymbol{\rho}_{\boldsymbol{X}}$ instead of the variancecovariance matrix $\boldsymbol{\Sigma}_{\boldsymbol{X}}$. Hence, in step 3 above we will calculate $\boldsymbol{\rho}_{\boldsymbol{X}}$ with elements

$$
\rho_{i j}=\left\{\begin{array}{lll}
1, & \text { for } & i=j \\
\frac{\sigma_{i j}}{\sigma_{i}^{2} \sigma_{j}^{2}} & \text { for } & i \neq j
\end{array}\right.
$$

and will perform the rest of the steps as mentioned above.

\section{Empirical Results for the PCA Dividend Factor Model}

\subsection{Construction of the Principal Components}

Generally speaking, high correlations between macroeconomic variables is not a problem in the PCA regression framework. (Therefore, we can include two or more macroeconomic variables that are highly correlated and use PCA regressions in order to exploit any extra information that is included in these additional variables.) Hence, we tried to construct Principal Components from an extensive and in many cases overlapping set of macroeconomic variables that can influence the magnitude of the dividends. Although there exists an extensive literature using macroeconomic factors that influence stock price valuation, it has rarely been done for dividends. In addition to the specific profits and earnings of the companies which are the primary forces of dividend valuation, there must be some other direct or indirect macroeconomic factors that influence dividend valuation in general. Moreover, according to many stock valuation models such as the expected discounted dividends model the price of the stock of a company is based on the magnitude of its dividends. Thus it might be also possible that the same factors that have been used extensively in the past to explain the prices of stocks can be used again to explain the magnitude of the dividends. Toward that end, we used two sets of original macroeconomic variables. The first case is the more exhaustive one. We have used 22 variables which could have influence dividend formation. Most of the variables in the first set are well documented in the financial literature from prominent academics to have influenced stock prices. However, in this set we have also included some other macroeconomic variables and indicators that possibly influence or predict dividend formation. In the second case, we have used a more parsimonious set of variables that were documented in the past to have an impact in stocks. 
Table 1: Glossary and Definitions of Variables (22 Macroeconomic Variables)

\begin{tabular}{|c|c|c|}
\hline Symbol & Variable & Definition or Source \\
\hline & \multicolumn{2}{|c|}{ Basic Series } \\
\hline I & Inflation & Log relative of U.S. Consumer Price Index \\
\hline $\mathrm{TB}$ & Treasury Bill Rate & $\begin{array}{l}\text { End-of-period return on 3-month bills; } \\
\text { FRED@ (Federal Reserve Economic Data) }\end{array}$ \\
\hline LGB & Long Term Government Bonds & 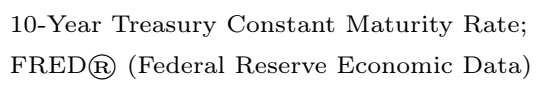 \\
\hline Baa & Low-grade bonds & $\begin{array}{l}\text { Return on Baa Rated bonds ; (Moody's } \\
\text { Seasoned Baa Corporate Yield) }\end{array}$ \\
\hline OS & Oil Prices (SPOT) & FRED@ (Federal Reserve Economic Data) \\
\hline $\mathrm{RPCE}$ & Real Personal Consumption Expenditures & FRED @ (Federal Reserve Economic Data) \\
\hline RPDI & Real Personal Disposable Income & FREDß) (Federal Reserve Economic Data) \\
\hline GDP & Gross Domestic Product (Growth) & FRED® (Federal Reserve Economic Data) \\
\hline $\mathrm{OP}$ & Output Productivity & U.S. Bureau of Labor Statistics \\
\hline IP & Industrial Production & $\begin{array}{l}\text { Industrial Production during Month; } \\
\text { FREDß(Federal Reserve Economic Data) }\end{array}$ \\
\hline UR & Unemployment Rate & U.S. Bureau of Labor Statistics \\
\hline REXP & Real Exports of Goods \& Services & U.S. Bureau of Economic Analysis \\
\hline NEXP & Real Net Exports & U.S. Bureau of Economic Analysis \\
\hline $\mathrm{CP}$ & Corporate Profits After Tax (Growth) & U.S. Bureau of Economic Analysis \\
\hline NDIV & Net Corporate Dividends (Growth) & U.S. Bureau of Economic Analysis \\
\hline MCSI & University of Michigan Inflation Expectation & $\begin{array}{l}\text { Survey Research Center: University of } \\
\text { Michigan }\end{array}$ \\
\hline \multirow[t]{2}{*}{ PMI } & Purchasing Managers Index & Institute for Supply Management \\
\hline & \multicolumn{2}{|c|}{ Derived Series } \\
\hline $\mathrm{YP}(t)$ & Annual Growth, Industrial production & $\ln [\operatorname{IP}(t) / \operatorname{IP}(t-12)]$ \\
\hline MOM & Momentum & $\begin{array}{l}\text { Fama \& French; (WRDS: Wharton Research } \\
\text { Data Services) }\end{array}$ \\
\hline SMB & Small minus Big & Fama \& French; (WRDS) \\
\hline HML & High minus Low & Fama \& French; (WRDS) \\
\hline INV & Real Gross Private Domestic Investment & U.S. Bureau of Economic Analysis \\
\hline $\operatorname{OPG}(t)$ & Output Productivity (Growth) & $\ln [\mathrm{OP}(t) / \mathrm{OP}(t-12)]$ \\
\hline OG & Oil Prices (Growth) & Annual growth on Real Prices \\
\hline MKT-RF & Risk Premium $(F \& F)$ & Fama \& French; (WRDS) \\
\hline $\operatorname{UTS}(t)$ & Term Structure & $\operatorname{LGB}(t)-\mathrm{TB}(t-1)$ \\
\hline $\operatorname{UPR}(t)$ & Risk Premium (C\&R\&R) & $\operatorname{Baa}(t)-\operatorname{LGB}(t)$ \\
\hline $\mathrm{RHO}(\mathrm{t})$ & Real Interest (ex post) & $\mathrm{TB}(t-1)-\mathrm{I}(t)$ \\
\hline
\end{tabular}

In the first set of variables we have included the Chen, Roll and Ross $(C \& R \& R)$ factors such as Real Interest Rate, Term Structure, Risk Premium, Industrial Production Growth, Inflation and Oil Prices Growth (see their paper ([4]) for more details). All of these factors were constructed using the methodology mentioned in that paper. It was not always possible for some of the basic series used in that paper to be obtained from the same sources. In such cases sensible alternative sources were used and series were obtained. ${ }^{14}$ In addition, we have included the three well known factors from Fama \& French (F\&F) ([10]) combined with the Momentum factor which is calculated and provided also from them. Moreover, following Cochrane

\footnotetext{
${ }^{13}$ An elbow means that after a specific eigenvalue the line in the plot is very flat in comparison with the previous one.

${ }^{14}$ For example, we used the 10-Year Treasury Constant Maturity Rate as the alternative for their Long Government Bonds variable which we obtained from FREDß) (Federal Reserve Economic Data).
} 
Table 2: Glossary and Definitions of Variables (7 Macroeconomic Variables)

\begin{tabular}{|c|c|c|}
\hline Symbol & Variable & Definition or Source \\
\hline & \multicolumn{2}{|c|}{ Basic Series } \\
\hline I & Inflation & Log relative of U.S. Consumer Price Index \\
\hline TB & Treasury Bill Rate & $\begin{array}{l}\text { End-of-period return on 3-month bills; } \\
\text { FRED@ (Federal Reserve Economic Data) }\end{array}$ \\
\hline LGB & Long Term Government Bonds & 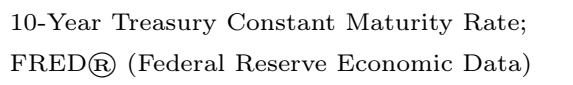 \\
\hline Baa & Low-grade bonds & $\begin{array}{l}\text { Return on Baa Rated bonds ; (Moody's } \\
\text { Seasoned Baa Corporate Yield) }\end{array}$ \\
\hline $\mathrm{RPCE}$ & Real Personal Consumption Expenditures & FRED® (Federal Reserve Economic Data) \\
\hline GDP & Gross Domestic Product (Growth) & FRED@ (Federal Reserve Economic Data) \\
\hline \multirow[t]{2}{*}{ IP } & Industrial Production & 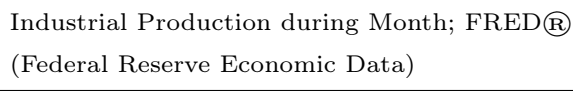 \\
\hline & \multicolumn{2}{|c|}{ Derived Series } \\
\hline $\mathrm{YP}(t)$ & Annual Growth, Industrial production & $\ln [\operatorname{IP}(t) / \operatorname{IP}(t-12)]$ \\
\hline INV & Real Gross Private Domestic Investment & $\begin{array}{l}\text { U.S. Department of Commerce: Bureau of } \\
\text { Economic Analysis }\end{array}$ \\
\hline $\mathrm{UTS}(t)$ & Term Structure & $\operatorname{LGB}(t)-\mathrm{TB}(t-1)$ \\
\hline $\operatorname{UPR}(t)$ & Risk Premium (C\&R\&R) & $\operatorname{Baa}(t)-\operatorname{LGB}(t)$ \\
\hline
\end{tabular}

$([5,6])$, we have included an investment and unemployment variable in our set of variables. Finally, we have included variables such as GDP growth, Real Personal Consumption Expenditures and Profits. Table 1 provides information about the whole set of original variables that were included in the PC calculation. ${ }^{15}$ We mention here two important points. The first point is that we have transformed each original variable, for example calculating growth from a basic series, in order to make every series stationary. Toward that end, we employed Dickey-Fuller Tests in order to test for any possible unit roots. The second point is that the variables are not enumerated under the same units of measurement. Therefore, we used the correlation matrix in the PC calculation instead of the covariance matrix. Use of the correlation matrix ensures the problem is overcome.

In the second set of variables we included only the $C \& R \& R$ factors that are found to be significant in their paper. In our case, we used only the observable inflation instead the unexpected and expected versions used in their seminal paper. In addition, we used GDP growth, Personal Consumption and Investment since it is the mainstream view that these particular variables could have a significant influence in the formation of the total dividends of a company. Table 2 provides information about this second set of original variables. ${ }^{16}$

In the calculation of Principal Components we used data from the years: 1963-2006. In the extensive case of 22 variables we used the first $15 \mathrm{PC}$ in our regressions. This choice is not only based on the eigenvalues but is also based on feedback from the regressions results. In addition, this number of PC explains most of the variation as it appears in the correlation matrix. In our case interpretation is difficult because we use a relatively large amount of companies and variables and therefore it is purposefully omitted. What is more important in our case is the normality assumption of the Principal Components as some of our simulations are directly based on that assumption as Result 3.10 indicates. Toward that end we have employed Q-Q plots in order to inspect whether there are some significant deviations from normality. Figure 1 provides a concise picture for the entire $22 \mathrm{PC}$. As we see in this figure there are only 3 or 4 out of $22 \mathrm{PC}$ that deviate substantially from the straight line. Therefore, normality tests have been employed for all $22 \mathrm{PC}$ in order

\footnotetext{
${ }^{15} \mathrm{~TB}, \mathrm{LGB}, \mathrm{Baa}, \mathrm{OS}, \mathrm{OP}$, IP variables were used only in order to calculate derived series and there were not used directly in the calculation of PC. The other 22 variables were used directly as input in the calculation of our PC.

${ }^{16} \mathrm{~TB}$, LGB, Baa, IP variables were used only in order to calculate derived series and there were not used directly in the calculation of PC. The other 7 variables were used directly as input in the calculation of our PC.
} 


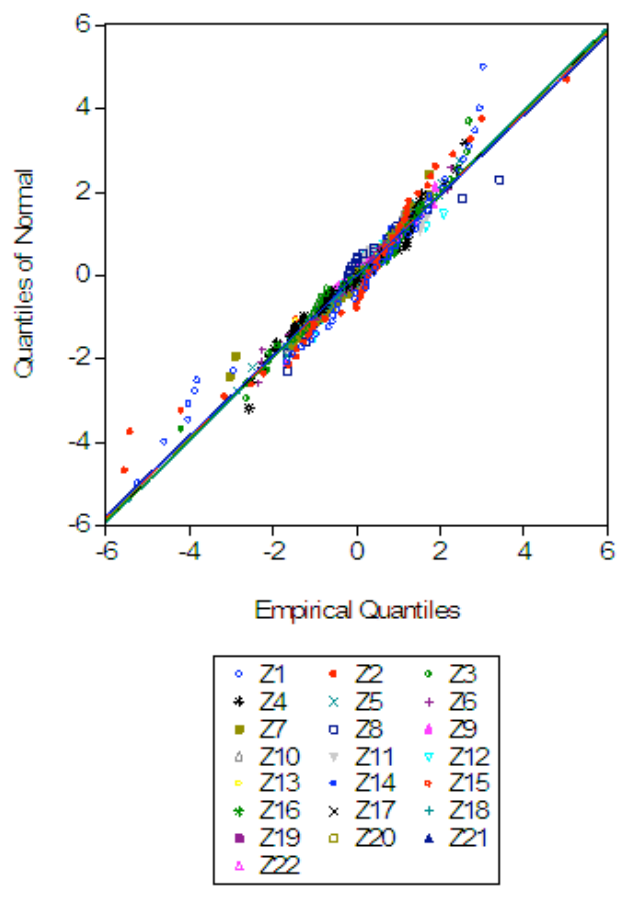

Figure 1: Q-Q Plot PC 22 Variables

to check the normality assumption. According to the Kolmogorov-Smirnov test we can reject the normality assumption only for the three following PC: $Z 2, Z 8, Z 11$. Moreover, in our case we need to check if the multivariate assumption indicated by Result 3.9 is valid. In practice, there are no "good" and reliable tests to examine joint normality for more than two dimensions. However, for most practical purposes, univariate and bivariate investigations are sufficient. For the bivariate case we have employed scatterplots for all the pairs of PC. Figure 2 provides all the pairwise combinations of scatterplots between PC with their respective confidence ellipses. One should immediately notice here that almost all of the points are inside of the ellipses which is an indication that these variables are jointly normal. Theoretically speaking, there always exist the following possibility: a particular data set appears normal in the first two dimensions but to have non-normal aspects in higher dimensions. However, such pathological data sets that appear normal in lower dimensions but are non-normal in higher dimensions are very rare in practical applications. ${ }^{17}$ We would like to also mention that our objective is not to prove beyond any doubt that our calculated PC are almost certain to be described by a joint normal distribution but rather to illustrate the point that the assumption that most of the PC are jointly normal is not entirely unrealistic. Thus, we believe that for theoretical simulation purposes the normality test results mentioned above are quite encouraging and sufficient for our particular case. Moreover, it is also important here to mention the fact that for more specific practical purposes one might also consider using various transformations in order to achieve normality of data (see section 4.8 in Johnson \& Wichern [17]).

In the calculation of Principal Components of the second case (7 variables) we used data from the years: 1962-2006. In this case, we keep all the PC based on feedback from the regression results. In this parsimonious second case, an interpretation was attempted based on the calculated eigenvectors mentioned in Table 3. Needless to say, this interpretation is quite subjective and not unique as always is the case in Principal Component Analysis. According to Table 3, the first PC loads heavily on GDP growth (GDP), industrial production (YP), investment (INV) and Consumption (RPCE). Therefore, we can interpret this variable as an "economy " or "expansion" factor. The second PC loads heavily on the Term Structure (UTS) and Risk Premium (URP) and it has considerable negative loads on both GDP and inflation (I). Thus, it

\footnotetext{
${ }^{17}$ For more details on this issue we refer the reader to see Johnson \& Wichern ([17]) chapter 4.
} 


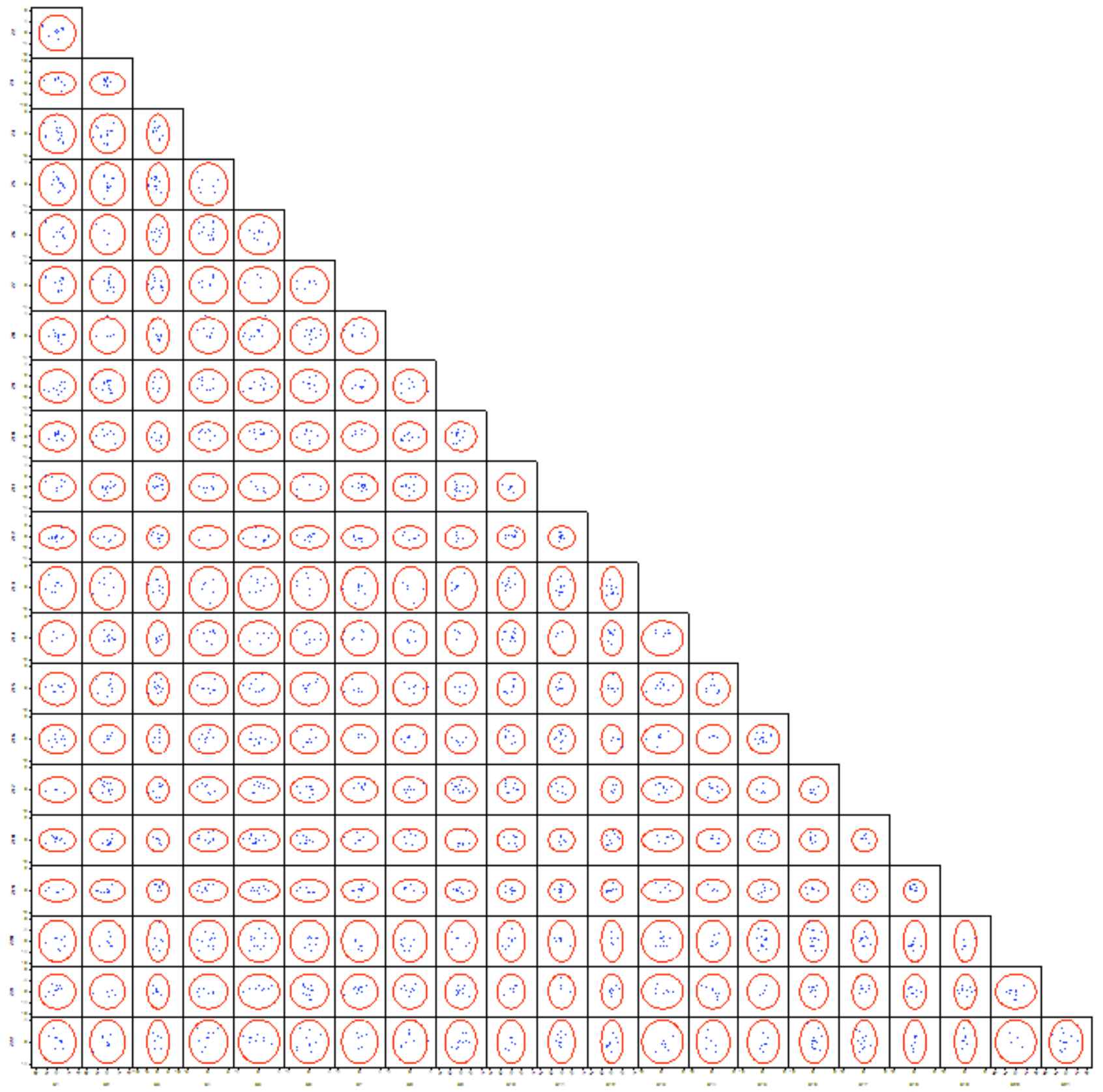

Figure 2: Scatterplots: Checking for Bivariate Normality (Lower Triangular Matrix 22 PC case)

can be considered as a "risk" or "recession" factor. The third PC loads heavily on INV and I variables and hence it can be considered as an "economy investment" factor. Although interpretation is quite difficult for the fourth factor one should notice the magnitude and the opposite loadings in UTS and UPR. This may be indicative that this PC is closely related with high long-term interest rates and thus it might be considered as an "anticipated inflation" factor. Similarly, an easy interpretation is also difficult for the fifth PC. This $\mathrm{PC}$ is characterized from a large loading in inflation and industrial production, an even larger loading in magnitude but opposite in sign for investment, neutral loading on GDP growth and a considerable loading in consumption. Those facts are probably describing an export of capital and thus the PC could be considered as "capital flight" or an "investment abroad" factor. The sixth PC loads heavily on consumption, has a very 


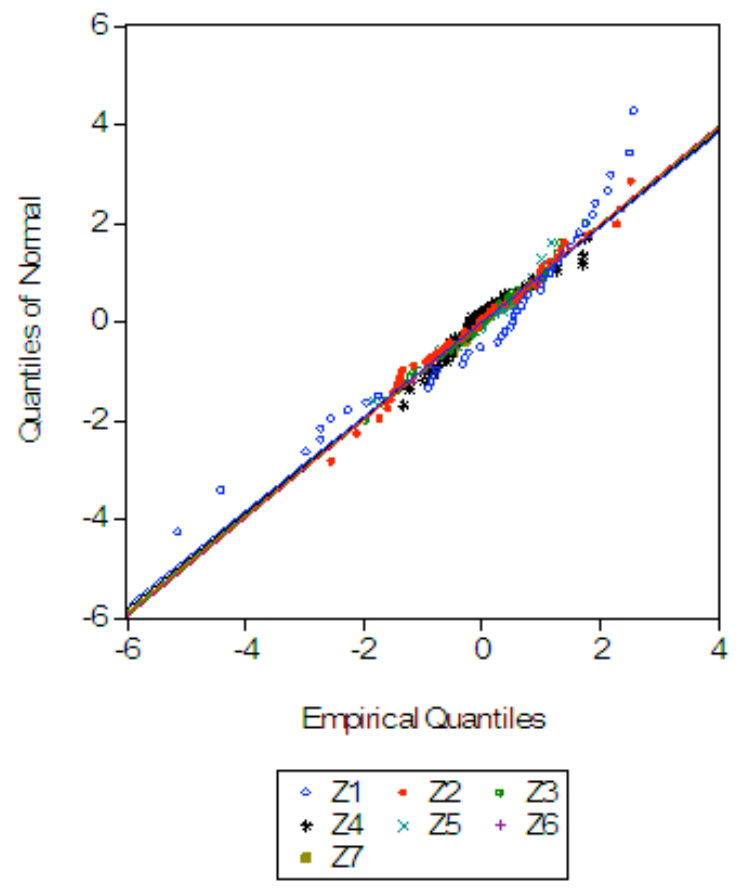

Figure 3: Q-Q Plot PC 7 Variables

large negative loading on industrial production. In addition, it has a considerable loading on GDP and a considerable negative loading on investment. Hence, we can interpret this $\mathrm{PC}$ as an "consumption-driven expansion" factor. Finally, the last PC has a small eigenvalue and we give no interpretation.

In a similar spirit with the first case mentioned above we test for the joint normality of all the PC starting from the univariate case. Figure 3 provides the Q-Q plot for all the PC combined in one graph. As this picture indicates one or two PC deviate considerably from their straight lines. According to KolmogorovSmirnov tests, $Z 1$ and $Z 4$ are probably not normal. However, all other variables pass the normality test. Finally, for the bivariate case, scatterplots for all the pairwise combinations of these seven PC have been employed and the corresponding graphs can be found in Figure 4. Similarly to the previous case, these scatterplots indicate that bivariate normality is not a totally unrealistic assumption given the limitations that are inherent in our specific data sets.

Table 3: Eigenvectors (loadings) 7 PC

\begin{tabular}{cccccccc}
\hline Variable & PC 1 & PC 2 & PC 3 & PC 4 & PC 5 & PC 6 & PC 7 \\
\hline UTS & 0.236 & 0.557 & 0.263 & -0.660 & 0.349 & 0.072 & -0.042 \\
UPR & -0.091 & 0.671 & 0.315 & 0.621 & -0.122 & 0.181 & -0.098 \\
GDP & 0.489 & -0.264 & 0.107 & 0.087 & 0.013 & 0.347 & -0.743 \\
YP & 0.460 & 0.054 & -0.059 & 0.326 & 0.409 & -0.713 & -0.012 \\
I & -0.273 & -0.392 & 0.744 & 0.100 & 0.444 & 0.032 & 0.101 \\
INV & 0.405 & -0.087 & 0.499 & -0.126 & -0.674 & -0.235 & 0.233 \\
RPCE & 0.497 & -0.076 & -0.116 & 0.199 & 0.213 & 0.526 & 0.610
\end{tabular}




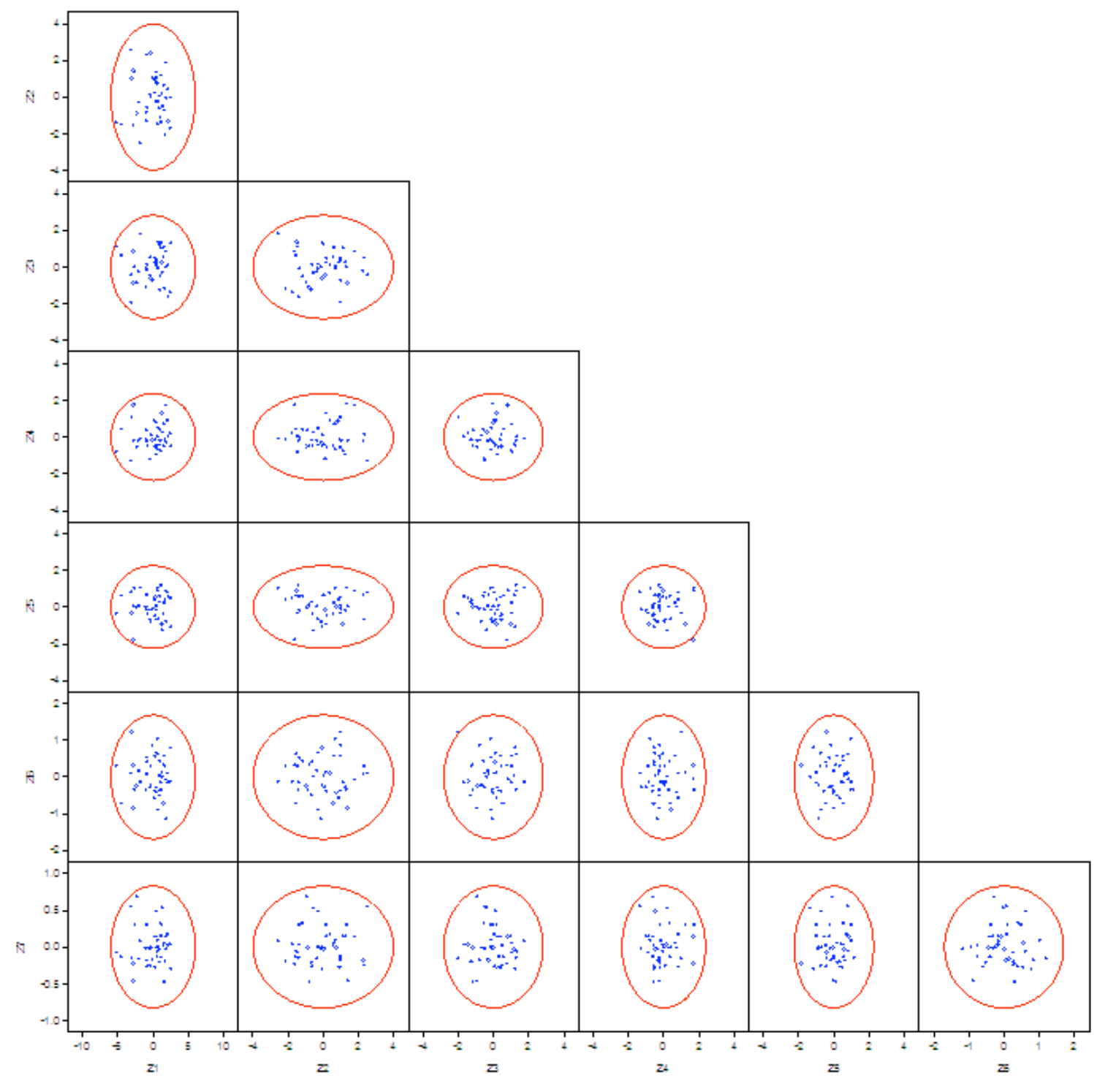

Figure 4: Scatterplots: Checking for Bivariate Normality (Lower Triangular Matrix 7 PC case)

\subsection{The Problem of Stationarity and Detrending the Data}

We already have mentioned the fact that we have transformed each original variable in order to make every series stationary. ${ }^{18}$ The implication was that the resulting PC were also stationary. We employed Dickey-Fuller Tests in order to test for any possible unit roots in any series that we have used in our analysis. According to those tests, all the macroeconomic variables were stationary after the appropriate transformation took place whenever this was necessary.

In addition, we collected the total dividends distributed by 33 companies for the years 1976-2006. ${ }^{19}$ This data was obtained from Standard \& Poor's Compustat@ Databases through Wharton Research Data Services (WRDS) access. Table 6 in the Appendix B provides the list with the names and symbols of these companies. We used the CPI in order to transform the dividends series in real terms for each company.

\footnotetext{
${ }^{18} \mathrm{By}$ the term stationarity we implicitly mean weak stationarity. For additional details see again Result 3.6 on page 10.

${ }^{19}$ This particular period was chosen due to the following reasons. First of all, availability is limited for this type of data. In addition, the quality of data deteriorates significantly in previous years due to splits, mergers, acquisitions, etc.
} 
Moreover, we applied the same detrending technique Shiller applied in his 1981 paper ([22]) to each company separately.

In the empirical implementation of the results, we note that 4 out of 33 companies (MCK, HAL, IBM and GM) appear to have a stationary log-dividend series after deflating them with the CPI. Moreover, the dividends data appears stationary according to plots and unit-root regressions after this particular method of detrending. We mention here that even after detrending we cannot reject the Dickey-Fuller Tests at $5 \%$ significant level for most of the companies. It is very probable that the reason according to which we cannot reject the null hypothesis of the unit root is that our sample is very small. However, plots and unit roots regressions for the vast majority of the companies suggest that the data series is stationary after this specific detrending takes place. Finally, pre-whitening of the series is applied to companies that have a significant autoregressive component.

\subsection{PCA Regression Results}

The final step in our PCA Dividends Factor Model was to estimate the coefficients $\xi_{i j}^{\prime}$ s for equation (3.5). Toward that end, we used the resulting calibrated PC and the series from the detrended total dividends of the companies as mentioned above. For every company we used any possible regression combination starting from the following initial regression equation

$$
\ln \left(d_{i}\right)=\alpha+\beta_{i 1} Z_{1}+\beta_{i 2} Z_{2}+\ldots+\beta_{i, 15} Z_{15}+\varepsilon_{i} .
$$

and we ended up in a regression equation of the form

$$
\ln \left(d_{i}\right)=\alpha+\mathbf{Z}_{\mathbf{M}} \boldsymbol{\beta}_{\boldsymbol{i} \boldsymbol{M}}+\varepsilon_{i}
$$

where $\mathbf{M}$ is a subset of integers $\{1,2, \ldots, 15\}$ such as the regression equation (4.1) has significant t-statistics for all of its regression coefficients. ${ }^{20}$ The following points are in order given the regression results. First of all, it seems that this particular detrending of the total dividends eliminates any possible heteroscedasticity problems. Several diagnostic tests for all of the regressions given by (4.1) did not show any significant problems except that some of the regressions showed some autocorrelation signs. However, any corrections that we used deteriorated substantially other aspects of the regressions and therefore were avoided. Generally speaking, the econometrically estimated results indicate that there is a significant relation between the dividends of stocks and the Principal Components constructed from various macroeconomic variables. $\bar{R}^{2}$ had a wide spectrum of values with many companies having a relatively large value (highest value being 0.75 ) indicating a high common-factor component.

Similarly, we calculated the regression coefficients for the case in which we used the variables mentioned in Table 2. Thus, for every company we used any possible regression combination starting from the following initial regression equation

$$
\ln \left(d_{i}\right)=\alpha+\beta_{i 1} Z_{1}+\beta_{i 2} Z_{2}+\ldots+\beta_{i, 7} Z_{7}+\varepsilon_{i} .
$$

and we ended up in a regression equation of the form

$$
\ln \left(d_{i}\right)=\alpha+\mathbf{Z}_{\mathbf{M}} \boldsymbol{\beta}_{\boldsymbol{i} \boldsymbol{M}}+\varepsilon_{i}
$$

where $\mathbf{M}$ is a subset of integers $\{1,2, \ldots, 7\}$ such as the regression equation (4.2) has significant $t$-statistics for all of its regression coefficients. The same general comments concerning the econometric aspects of the regressions apply also for this case as mentioned above. The main difference in this case is that the variation explained was lower as measured by both $\bar{R}^{2}$ and the t-statistics of the regressions coefficients. The reason is that we employed fewer macroeconomic variables and thus fewer Principal Components in the regression equation.

\footnotetext{
${ }^{20} \mathbf{Z}_{\mathbf{M}}$ is a row vector and $\boldsymbol{\beta}_{i M}$ is a column vector. The elements in both vectors correspond to the number of elements in $\mathbf{M}$.
} 


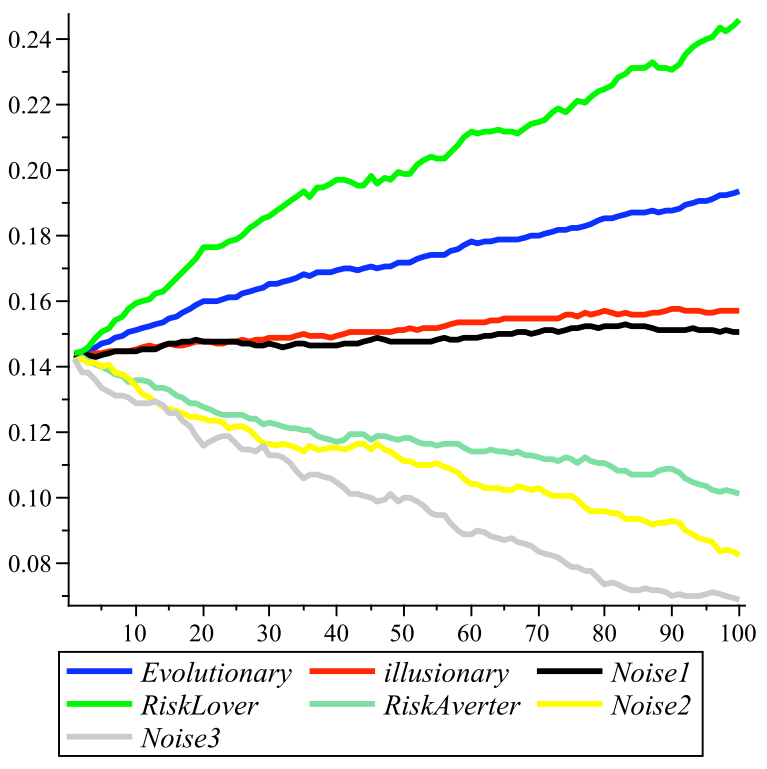

(a) 100 Time Periods.

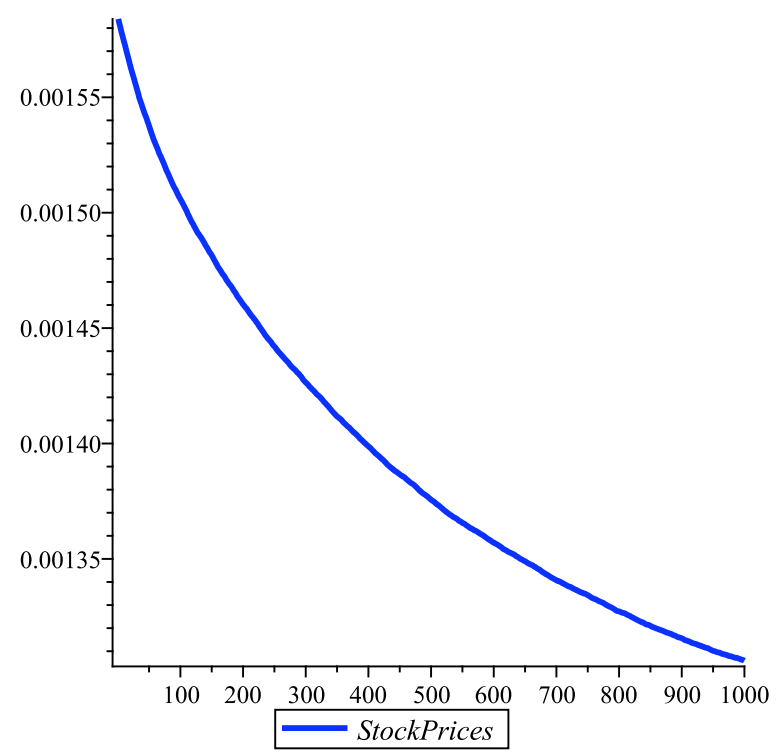

(b) A Typical Relative Price for this Case.

Figure 5: $\lambda^{*}$ vs $\lambda^{R L}$ vs $\lambda^{i l l u}$ with randomly generated dividends. The typical scenario in this case is that $\lambda^{R L}$ performs better than $\lambda^{*}$ even in the first 100 time periods. The typical relative stock price path is relatively smooth.

\section{Simulation Results}

\subsection{Empirical Results for the Evolutionary Portfolio Theory}

In two papers $([14,15])$, Hens, et. al. apply their evolutionary portfolio model discussed in Section 2 to a real total dividends data from both the Wall Street and Swiss Exchange Market. Their motivation is to test the theoretical results of the evolutionary portfolio rule in a less idealized situation where long-lived assets and incomplete markets are considered. In order for this empirical investigation to be possible, the authors restrict their attention only to fixed-mix rules, i.e. the exact model that is mentioned in Section 2. A fixed-mix rule (or rebalancing rule) holds certain portfolio weights constant for a long period. Hence, if market prices fluctuate, a rebalancing rule has to adjust the number of shares it holds in order to keep the proportions of wealth assigned to specific stocks constant. Fernholz and Maguire ([12]) consider fixed-mix rules in a different context ${ }^{21}$ where they argue that fixed-mix strategies are quite profitable and capture the volatility that is present in stock markets in a natural way.

These authors considered rules from both the Behavioral and the Traditional Finance approach and employed strategies such as the Cumulative Prospect Theory, the Mean-Variance, the Conditional Valueat-Risk (CVaR) and the Growth Optimal Rule (GOP) among others. Significantly enough, among those rules is also the so called Illusionary Diversification Rule from Benartzi and Thaler ([2]) which assigns the same share for all assets and this is exactly the reason why it is called illusionary. Illusionary or not, this strategy is explored in Fernholz and Maguire ([12]) where they have shown that, in the absence of trading commissions, such portfolios can generate remarkably high information ratios. The main result of Hens, et. al. $([15,14])$ is that the Evolutionary Portfolio Rule $\lambda^{*}$, as given by $(2.16)$, is the winner of the contest independently of the initial share of the total market wealth that it was given to this rule. In every case, $\lambda^{*}$ was able to dominate the total market wealth after a relatively small number of periods.

In the empirical implementation of the Evolutionary Portfolio Theory mentioned above, there are many limitations concerning both the quality and the quantity of a particular data-set. Thus there is a substantial motive to repeat the simulations using randomly generated dividend data-sets. In that way, there is no limit

\footnotetext{
${ }^{21}$ Employing the concepts of Stochastic Portfolio Theory and Excess Growth Rates.
} 
in the number of companies and time periods for acquiring dividends data. Therefore, the empirical results will be more robust than before.

\subsection{The First Set of Results}

In our first test set of simulations, we use five strategies including the Evolutionary Portfolio Rule given by:

$$
\lambda_{k}^{*}=\left(1-\lambda_{0}\right) \mathbb{E} d^{k}(s), \quad k=1,2, \ldots, K
$$

where $d^{k}=\frac{D^{k}}{D}$ denotes the relative dividend distributed by company $k \cdot{ }^{22}$ The major opponent of that rule in that particular set is the illusionary diversification rule given by:

$$
\lambda_{k}^{i l l u}=\frac{\left(1-\lambda_{0}\right)}{K}, \quad k=1,2, \ldots, K .
$$

Fernholz and Maguire ([12]) propose the idea that weights on more volatile stocks could be increased to generate greater "excess growth," or decreased in order to control portfolio risk while still applying fixed-mix strategies. Therefore, having that context in mind we developed a further two strategies, with the first one trying to capture increased risk and thus is named "Risk Lover." More explicitly:

$$
\lambda_{k}^{R L}=\left(1-\lambda_{0}\right) \sigma_{d_{k}}^{*}
$$

where $\sigma_{d_{k}}^{*}$ is the corresponding normalized standard deviation of the relative dividend. The other is trying to capture lower risk so:

$$
\lambda_{k}^{R A}=\left(1-\lambda_{0}\right)\left(\frac{1}{\sigma_{d_{k}}}\right)^{*}
$$

where $\left(\frac{1}{\sigma_{d_{k}}}\right)^{*}$ is the normalized inverse of the corresponding standard deviation of the relative dividend and hence is named "Risk Averter." In addition, we use three noise-trading rules that assign weights according to some particular distributions. Furthermore, we have assumed that the dividends process is lognormal across companies, which is a reasonable assumption according to the empirical literature. All portfolio strategies considered here devote the same proportion of wealth in consumption according to the "equal saving" rationale and it is assumed to be $10 \%$ of the total wealth i.e $\lambda_{0}=0.1$.

In figure 5 one can find the diagram for the result of the simulations including the above mentioned strategies. The surprising fact here is that although $\lambda^{*}$ is doing relatively well from the first year, $\lambda^{R L}$ is doing even better for the first 100 time periods! Eventually $\lambda^{*}$ dominates after a sufficient number of time periods.

As an important remark here, we need to mention the point that the above mentioned diagrams are consistent with the stability and instability criteria given by Theorem 2.7 in section 2 . The practical illustration of these theorems is given by the diagram (b) in figure 6 . This diagram clearly illustrates the point that $\lambda^{*}$ is the winner in a contest between these two strategies. "Risk Lover" $\left(\lambda^{R L}\right)$ seems to be the winner for the first time periods in a contest whereas other strategies also exist. In a sense, $\lambda^{R L}$ exploits the "irrationality" of other strategies better and thus it earns better profits in a short time period even in comparison with the true "smart" and fundamental rule which is $\lambda^{*}$. This is one of the main points that makes Evolutionary Portfolio Theory very appealing as an economic theory. It can describe, in a theoretical way, what is really happening in financial markets accommodating the fundamentals of the mainstream financial theory with other more alternative views of the financial markets such as the Behavioral Finance approach.

\subsection{Simulations with The Calibrated Model}

According to the mathematical results of section 2, there is a strong indication that the Evolutionary Rule $\lambda^{*}$ developed by Hens and Schenk-Hoppé ([16]) is the most probable long run survivor of any evolutionary financial competition. However, as much as asymptotic behavior is extremely important for the evolution of a general theory there is also the need for results which try to explain phenomena that are shorter in time.

\footnotetext{
${ }^{22}$ The term in the parenthesis $\left(1-\lambda_{0}\right)$ is simply an adjustment for consumption.
} 


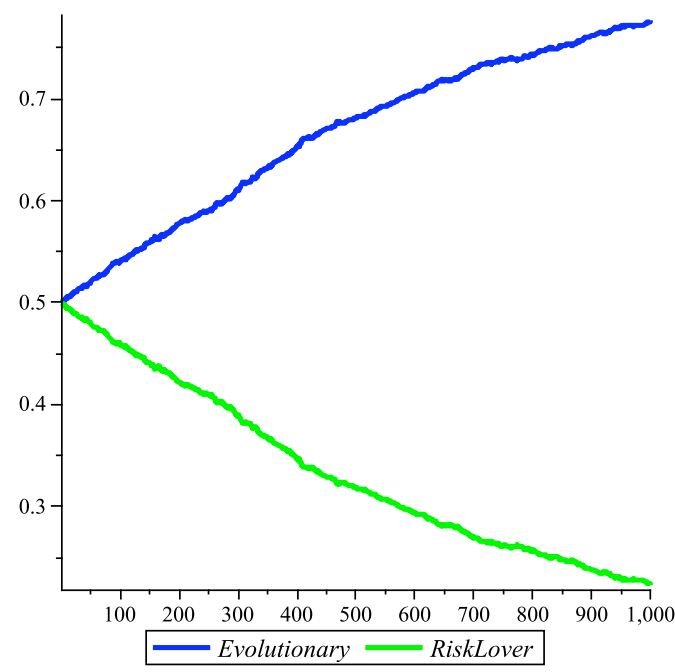

(a) Equal Initial Weights.

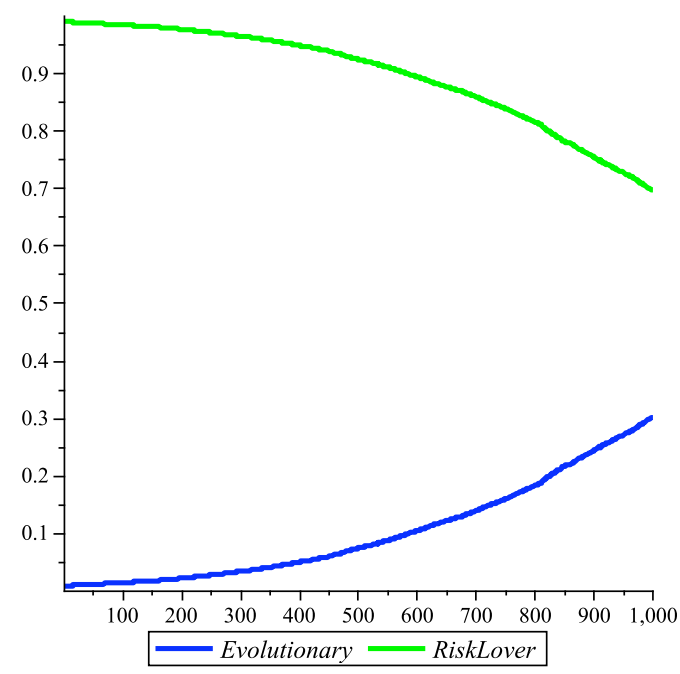

(b) Evolutionary Rule with Initial Comparative Disadvantage.

Figure 6: $\lambda^{*}$ vs $\lambda^{R L}$ in a competition between them.

As we mentioned above there is a wide spectrum of results which depends mainly on the available strategies that consists in every case the "ecosystem" of the market. We have seen that in case where we have used various random generated dividends data sets, it is also possible for other fixed-mix strategies than $\lambda^{*}$ to perform better even in more than 100 time periods. ${ }^{23}$ Thus, the need to perform some sets of simulations using some calibrated dividend data sets in order to test the possibility of whether a fixed-mix strategy could perform better than $\lambda^{*}$ in the short run was evident. Therefore, in this section we provide simulations results using dividends data sets arising from the PCA Dividend Factor Model developed in section 3.3 which has been calibrated in section 4 . Finally, we need to mention that for all sets of simulations for this section we substituted the "Noise" strategies with the following one:

$$
\lambda_{k}^{A}=\left(1-\lambda_{0}\right) \frac{a_{k}}{\sum_{j} a_{j}}
$$

which was called strategy "Alfa" since it is based on the estimated constants of the regressions. This strategy was used as the "behavioral" improvement of the naive diversification strategy $\lambda^{i l l u}$ and it performed better than $\lambda^{i l l u}$ in every set of simulations that is mentioned in this section.

Figure 7 provides the results of the simulations for the PCA Dividend Factor Model given by (3.5) and the regression results from our calibration procedure. In this typical set of simulations, we see that $\lambda^{*}$ does not face any serious threat from any other strategy and conquers most of the available wealth from the first 100 time periods. These results are in line with the results of Hens et al. ([14, 15]) except for a very important fact. Our case indicates a lot more volatility than in their case as diagram (b) in figure 7 clearly indicates. ${ }^{24}$ This is an important aspect of the dividend factor model in combination with this particular set of strategies and it is not attributed to any particular "noise trading" rules. This is a desirable aspect of the results in light of the infamous debate around the "excess volatility" of stock prices initiated by the work of Shiller ([22]). Figure 8 provides the results of the $7 \mathrm{PC}$ calibrated case. The results are similar as in the previous calibrated case except for the fact that volatility seems to be even more severe in this case.

Tables 4 and 5 in Appendix B provide the calculated weights of the corresponding five strategies to the

\footnotetext{
${ }^{23}$ In some extreme cases, even more than 10,000 time periods.

${ }^{24}$ Hens et al. ([14]) provide a case with volatile prices but due to the existence of a particular noise strategy. This is the main reason that we eliminated any "Noise Traders" strategies from this set of simulations since we wanted to illustrate that volatility in our case is not solely arising from the existence of any "Noise Traders" Strategies.
} 


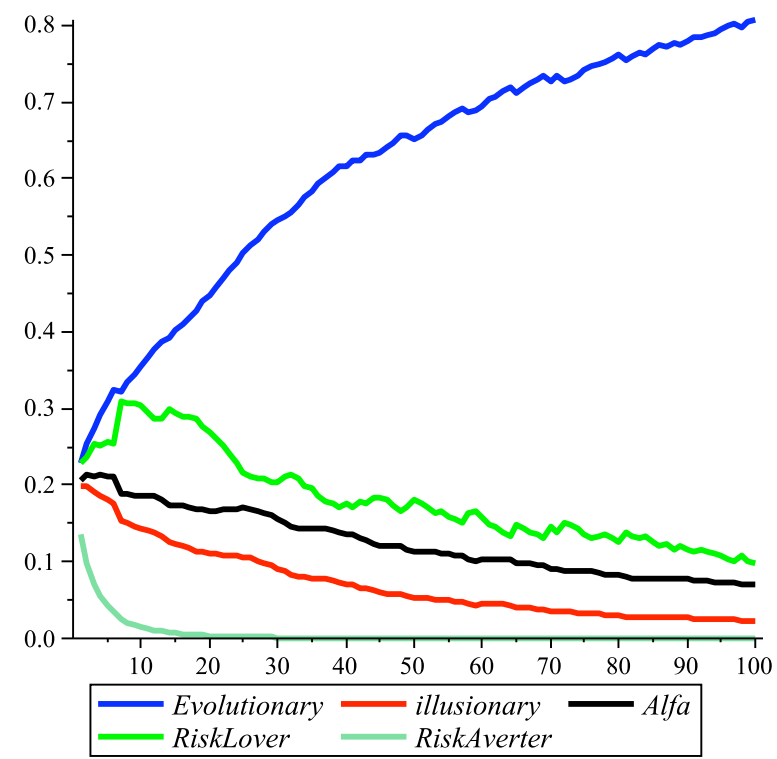

(a) A Typical Simulation for 100 Time Periods.

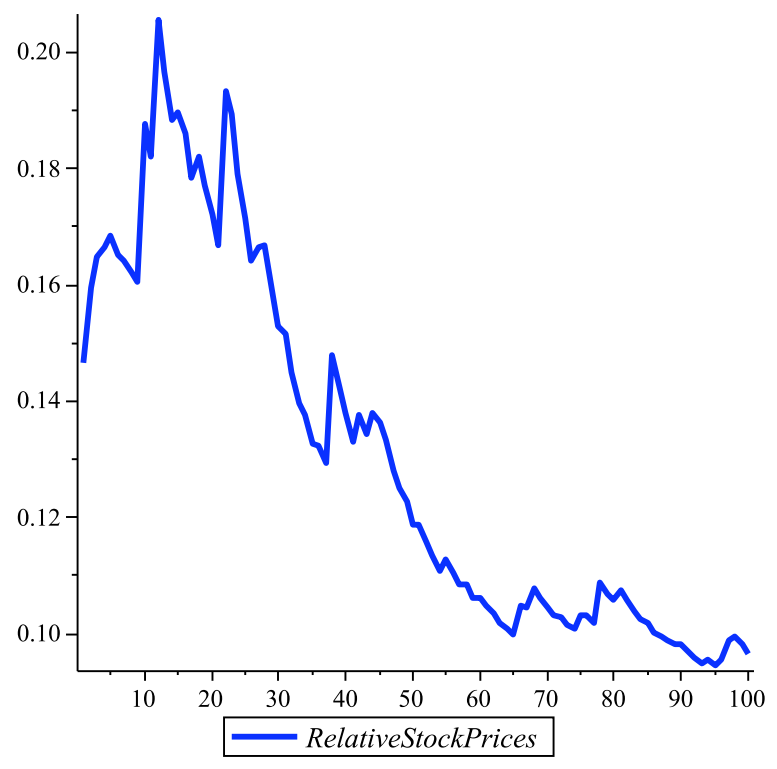

(b) A Typical Relative Price Path for 100 Time Periods.

Figure 7: Calibrated Model (22 Variables). Notice that the typical stock price path is a lot more volatile than the previous case.

set of simulations mentioned here. ${ }^{25}$

\subsection{Some Intuition about the Results}

In this part we will try to explain briefly the reasons for the significance of the results mentioned in this section. Toward that end, we need to recall some things about Evolutionary Finance. Traditional finance models based on fully rational economic agents are theoretically elegant and appealing, but they did not succeed empirically in the past. Thus we need a theoretical model that explains well what happens in real life without throwing out all the useful traditional finance models that various economists have discovered so far. Evolutionary Finance, as discovered through the seminal work of Hens, et. al. ([16, 9, 8]), combines all available financial theories and provides a unique synthesis that explains all financial phenomena that we have observed in the recent past. It provides strong mathematical results that satisfy adequately the mainstream economic theories of traditional finance models. In addition, it provides a simulations framework, or evolutionary laboratory, in terms of which can analyze important phenomena and approximate real-life situations. In two papers, Hens, Schenk-Hoppé, and Stalder ([14]) as well as Hens and Schenk-Hoppé ([15]) open the way for a completely new empirical framework in economics using this evolutionary set-up and performing simulations with real dividends data. Their simulations produced striking results, among them the fact that the evolutionary rule $\lambda^{*}$ is the only survivor even if in shorter time periods there is some room for other rules to succeed.

It therefore, was desirable to perform a new set of simulations that would explore some interesting financial phenomena and provide some different methodological procedures. We have used randomly generated dividends via Monte Carlo simulations to test the results' variability for a large number of stocks under different distributions for the dividend process. The results were promising, for under some circumstances an "irrational" strategy can perform better than $\lambda^{*}$ even for a large number of periods.

In addition, one of the real-life financial phenomena is the excess volatility puzzle first mentioned by Robert Shiller ([22]), who discovered that stock prices are extremely volatile in comparison with the volatility of their corresponding dividend payments. Hens, Schenk-Hoppé and Stalder ([14]) cite a case where

\footnotetext{
${ }^{25}$ All strategies are diversified. The value of $0.0 \%$ that appears in many cases results from rounding and corresponds to a very small value.
} 


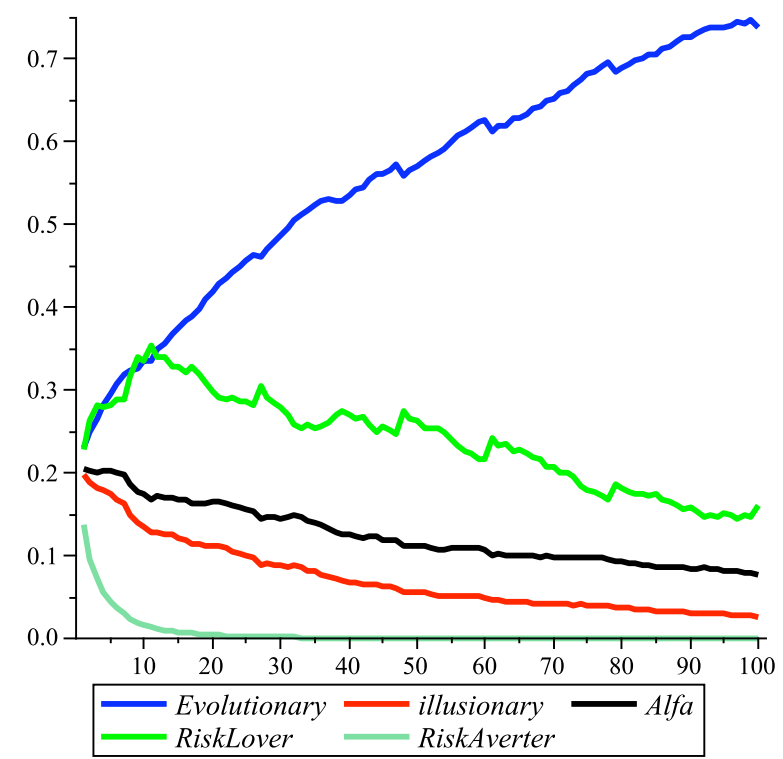

(a) A Typical Scenario for 100 Time periods.

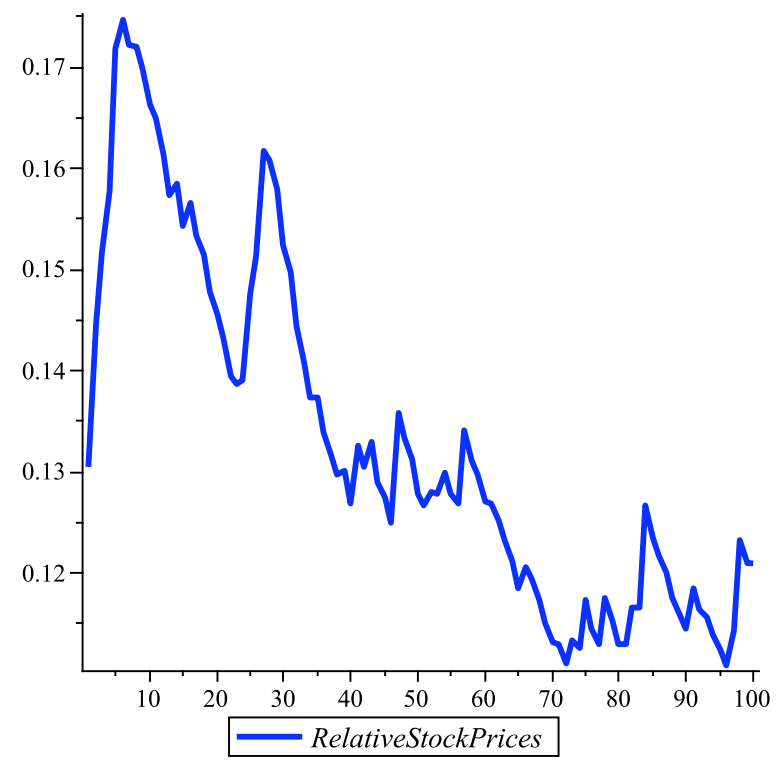

(b) A Typical Relative Price Path for 100 Time Periods.

Figure 8: Calibrated Model (7 Variables). Typical results, prices are even more excessively volatile.

"excess volatility" is generated within an evolutionary framework due to some noise trading. Moreover, many prominent economists ${ }^{26}$ have argued that managers tend to smooth the dividend process and that this tendency makes the issue problematic. Whatever the effect of dividend smoothing on the excess volatility issue may be, it is a fact worth considering. ${ }^{27}$ Because of this factor, we were motivated to introduce a dividend model that could generate some excess volatility into the evolutionary model and connect all dividends with common macroeconomic variables. Thus, in section 3.2 we provide a dividend model that reflects the theoretical aspects of the evolutionary model and satisfies the necessary assumptions in order theorem 2.7 to be valid. Simulated dividends generated according to this model are free from any smoothing effect, and the section above deals with corresponding simulations. We have calibrated our dividend factor model in the subsection above using data on real dividends and macroeconomic variables. We think that our particular mode of calibration suggests new approaches to empirical work. We have performed a set of simulations with artificial dividends based on our new dividends model that reflects the theoretical aspects of the evolutionary model but calibrated by using real data. In this case the highlight was that excess volatility was generated among the relative prices although $\lambda^{*}$ is the clear winner of the contest. ${ }^{28}$

Our results should come as a useful addition to the already existing results of the literature for both theoretical and practical reasons. In theoretical terms we reproduced an economy with a lot of real-life aspects built on the solid evolutionary finance framework developed by Hens \& Schenk-Hoppé ([16]). In addition, we produced economies that support the notion of the profitability for fixed-mix rules in financial markets. In practical terms we enhanced their results by suggesting new fixed-mix strategies that could be superior for investors in the short run.

\section{Conclusion}

We have explored the impact of various constant-proportions investment strategies in an evolutionary market in which dividends have been generated according to a new Dividend Factor Model. Moreover, we have performed a numerical analysis to study the long-run outcome of an evolutionary competition with several

\footnotetext{
${ }^{26}$ For an example, see Marsh \& Merton ([20]).

${ }^{27}$ Hens \& Schenk-Hoppé ([15]) mention a similar effect in their empirical work.

${ }^{28}$ See ([21]) for a particular case where an irrational strategy performs better than $\lambda^{*}$.
} 
well diversified constant-proportions strategies, among them some innovative strategies. We have presented and compared simulations with dividends being artificially generated according to our particular model.

Our initial motivation was to extend and enhance the existing evolutionary results of the literature. Toward that end we invented a new framework to connect dividends with macroeconomic variables utilizing statistical concepts from multivariate analysis. The statistical calibration of this model revealed many significant empirical aspects, a topic that deserves its own distinctive research. In our PCA Regression case the econometrically estimated results indicate that there is a significant relation between the dividends of stocks and the Principal Components constructed from various macroeconomic variables. These results support the use of PCA Regression analysis as an alternative solution to the multicollinearity problem.

We have improved and extended the evolutionary framework with various sets of Monte Carlo simulations. We used a different set of strategies since the practicability and interpretation of the results depend highly on the participating set of strategies. We have used only well diversified fixed-mix rules because any undiversified strategies are severely penalized according to this evolutionary set-up. Our results in the first set of simulations indicated that under some circumstances an "irrational" strategy could perform better than the Evolutionary Portfolio Rule for a significant number of periods. Moreover, the Evolutionary Portfolio Rule managed to asymptotically acquire the total wealth of the market independently of its initial market share. Therefore, although a theorem that guarantees global stability in this evolutionary market does not exist yet, our simulations support the notion, suggested by local growth rates, that this evolutionary rule behaves as being asymptotically globally stable. Our results are extremely important for both theoretical and practical reasons. In theoretical terms we have a model where, although the true rational strategy is the only probable stable strategy, it is also possible for some "behavioral" rules to perform better under some specific circumstances. In practical terms, we have for the very first time an outcome that clearly suggests that some irrational strategies could be extremely profitable for investors in an evolutionary market for a long time.

In addition, we have performed another set of simulations where we have tested the impact of the Dividend Factor Model in the evolutionary competition. This part of the simulations included dividends generated according to the calibrated estimates of our dividend model. We discovered that in our simulated economy there was a large amount of price volatility, although the Evolutionary Portfolio Rule was the clear winner of the contest. More significantly, in this particular "ecosystem" the volatility is not only very large but also very persistent over time.

Finally, the results from our particular simulation suggest avenues for future research. First, as just mentioned, a new evolution of wealth function could be developed in order to theoretically justify a large variety of fixed-mix rules. On the practical side, new strategies could be explored and their performances compared. In addition, transaction costs could be incorporated into the model and their effect measured by additional simulation studies. It is possible that this addition to the model will benefit strategies that avoid frequent trading. Additional strategies appropriate for this case should be developed and carefully tested. Last but not least, further research could explore and modify this general set-up in order to analyze similar phenomena in foreign exchange markets.

\section{Acknowledgments}

We would like to express our appreciation to Paul Beaumont for his valuable advice and remarks. 


\section{A Tables}

Table 4: Portfolio Weights (22 Variables)

\begin{tabular}{cccccc}
\hline Symbol & $\lambda^{*}$ & $\lambda^{\text {illu }}$ & $\lambda^{A}$ & $\lambda^{R L}$ & $\lambda^{R A}$ \\
\hline AA & $0.7 \%$ & $2.7 \%$ & $2.5 \%$ & $0.0 \%$ & $0.1 \%$ \\
AXP & $1.7 \%$ & $2.7 \%$ & $3.0 \%$ & $0.1 \%$ & $0.0 \%$ \\
AIG & $0.1 \%$ & $2.7 \%$ & $1.3 \%$ & $0.0 \%$ & $16.0 \%$ \\
BA & $0.9 \%$ & $2.7 \%$ & $2.6 \%$ & $0.0 \%$ & $0.1 \%$ \\
CAT & $1.1 \%$ & $2.7 \%$ & $2.7 \%$ & $0.1 \%$ & $0.0 \%$ \\
CVX & $5.0 \%$ & $2.7 \%$ & $3.6 \%$ & $0.8 \%$ & $0.0 \%$ \\
DIS & $0.2 \%$ & $2.7 \%$ & $1.4 \%$ & $0.0 \%$ & $0.3 \%$ \\
DD & $5.1 \%$ & $2.7 \%$ & $3.6 \%$ & $0.5 \%$ & $0.0 \%$ \\
GE & $4.9 \%$ & $2.7 \%$ & $3.6 \%$ & $0.4 \%$ & $0.0 \%$ \\
GM & $9.7 \%$ & $2.7 \%$ & $4.0 \%$ & $3.3 \%$ & $0.0 \%$ \\
HAL & $1.0 \%$ & $2.7 \%$ & $2.7 \%$ & $0.0 \%$ & $0.1 \%$ \\
MER & $0.3 \%$ & $2.7 \%$ & $2.0 \%$ & $0.0 \%$ & $2.3 \%$ \\
BAC & $0.1 \%$ & $2.7 \%$ & $1.1 \%$ & $0.0 \%$ & $51.0 \%$ \\
PFE & $0.6 \%$ & $2.7 \%$ & $2.4 \%$ & $0.0 \%$ & $0.2 \%$ \\
JPM & $0.3 \%$ & $2.7 \%$ & $2.1 \%$ & $0.0 \%$ & $1.7 \%$ \\
HPQ & $0.1 \%$ & $2.7 \%$ & $1.6 \%$ & $0.0 \%$ & $12.0 \%$ \\
HON & $0.8 \%$ & $2.7 \%$ & $2.5 \%$ & $0.0 \%$ & $0.2 \%$ \\
EMR & $0.9 \%$ & $2.7 \%$ & $2.6 \%$ & $0.0 \%$ & $0.2 \%$ \\
PEP & $0.8 \%$ & $2.7 \%$ & $2.6 \%$ & $0.0 \%$ & $0.2 \%$ \\
MO & $1.4 \%$ & $2.7 \%$ & $2.9 \%$ & $0.0 \%$ & $0.1 \%$ \\
COP & $1.8 \%$ & $2.7 \%$ & $3.0 \%$ & $0.2 \%$ & $0.0 \%$ \\
PG & $2.2 \%$ & $2.7 \%$ & $3.2 \%$ & $0.1 \%$ & $0.0 \%$ \\
MRK & $1.2 \%$ & $2.7 \%$ & $2.8 \%$ & $0.0 \%$ & $0.1 \%$ \\
XOM & $19.0 \%$ & $2.7 \%$ & $4.4 \%$ & $8.4 \%$ & $0.0 \%$ \\
KO & $2.1 \%$ & $2.7 \%$ & $3.1 \%$ & $0.1 \%$ & $0.0 \%$ \\
F & $9.8 \%$ & $2.7 \%$ & $3.5 \%$ & $67.0 \%$ & $0.0 \%$ \\
IBM & $12.0 \%$ & $2.7 \%$ & $4.1 \%$ & $8.8 \%$ & $0.0 \%$ \\
JNJ & $0.9 \%$ & $2.7 \%$ & $2.6 \%$ & $0.0 \%$ & $0.3 \%$ \\
MCD & $0.2 \%$ & $2.7 \%$ & $1.7 \%$ & $0.0 \%$ & $2.2 \%$ \\
MMM & $3.0 \%$ & $2.7 \%$ & $3.3 \%$ & $0.2 \%$ & $0.0 \%$ \\
RTN & $0.6 \%$ & $2.7 \%$ & $2.4 \%$ & $0.0 \%$ & $0.3 \%$ \\
UTX & $0.8 \%$ & $2.7 \%$ & $2.6 \%$ & $0.0 \%$ & $0.3 \%$ \\
MCK & $0.3 \%$ & $2.7 \%$ & $2.1 \%$ & $0.0 \%$ & $1.2 \%$
\end{tabular}


Table 5: Portfolio Weights (7 Variables)

\begin{tabular}{cccccc}
\hline Symbol & $\lambda^{*}$ & $\lambda^{\text {illu }}$ & $\lambda^{A}$ & $\lambda^{R L}$ & $\lambda^{R A}$ \\
\hline AA & $0.8 \%$ & $3.6 \%$ & $3.3 \%$ & $0.0 \%$ & $0.2 \%$ \\
AXP & $2.0 \%$ & $3.6 \%$ & $3.9 \%$ & $0.1 \%$ & $0.1 \%$ \\
AIG & $0.1 \%$ & $3.6 \%$ & $1.7 \%$ & $0.0 \%$ & $23.3 \%$ \\
BA & $1.0 \%$ & $3.6 \%$ & $3.4 \%$ & $0.0 \%$ & $0.2 \%$ \\
CAT & $1.2 \%$ & $3.6 \%$ & $3.5 \%$ & $0.3 \%$ & $0.0 \%$ \\
CVX & $6.1 \%$ & $3.6 \%$ & $4.8 \%$ & $0.8 \%$ & $0.0 \%$ \\
KO & $2.4 \%$ & $3.6 \%$ & $4.1 \%$ & $0.1 \%$ & $0.0 \%$ \\
EMR & $1.1 \%$ & $3.6 \%$ & $3.5 \%$ & $0.0 \%$ & $0.3 \%$ \\
XOM & $22.9 \%$ & $3.6 \%$ & $5.7 \%$ & $14.2 \%$ & $0.0 \%$ \\
GE & $8.4 \%$ & $3.6 \%$ & $4.7 \%$ & $53.7 \%$ & $0.0 \%$ \\
GM & $5.5 \%$ & $3.6 \%$ & $5.3 \%$ & $0.6 \%$ & $0.0 \%$ \\
HAL & $12.7 \%$ & $3.6 \%$ & $3.5 \%$ & $8.1 \%$ & $0.0 \%$ \\
HON & $1.2 \%$ & $3.6 \%$ & $3.3 \%$ & $0.0 \%$ & $0.1 \%$ \\
HPQ & $0.9 \%$ & $3.6 \%$ & $2.0 \%$ & $0.0 \%$ & $0.2 \%$ \\
IBM & $0.2 \%$ & $3.6 \%$ & $5.3 \%$ & $0.0 \%$ & $10.5 \%$ \\
JNJ & $12.8 \%$ & $3.6 \%$ & $3.4 \%$ & $11.5 \%$ & $0.0 \%$ \\
MCK & $1.0 \%$ & $3.6 \%$ & $2.8 \%$ & $0.0 \%$ & $0.3 \%$ \\
MER & $0.4 \%$ & $3.6 \%$ & $2.6 \%$ & $0.0 \%$ & $1.8 \%$ \\
MMM & $0.4 \%$ & $3.6 \%$ & $4.4 \%$ & $0.0 \%$ & $3.1 \%$ \\
BAC & $3.6 \%$ & $3.6 \%$ & $1.4 \%$ & $0.2 \%$ & $0.0 \%$ \\
PEP & $0.1 \%$ & $3.6 \%$ & $3.4 \%$ & $0.0 \%$ & $48.5 \%$ \\
PFE & $0.9 \%$ & $3.6 \%$ & $3.2 \%$ & $0.0 \%$ & $0.4 \%$ \\
MO & $0.7 \%$ & $3.6 \%$ & $3.8 \%$ & $0.0 \%$ & $0.6 \%$ \\
COP & $1.7 \%$ & $3.6 \%$ & $3.9 \%$ & $0.1 \%$ & $0.1 \%$ \\
RTN & $2.0 \%$ & $3.6 \%$ & $3.2 \%$ & $0.1 \%$ & $0.1 \%$
\end{tabular}

Table 6: List of Companies \& Symbols

\begin{tabular}{cccc}
\hline \hline Symbol & Name & Symbol & Name \\
\cline { 2 - 2 } AA & Alcoa, Inc. & EMR & Emerson Electrical \\
AXP & American Express & PEP & Pepsico \\
AIG & American International Group, Inc. & MO & Altria Group Inc. \\
BA & Boeing Co. & COP & ConocoPhillips \\
CAT & Caterpillar Inc. & PG & Proctor \& Gamble \\
CVX & Chevron & MRK & Merck Co Inc. \\
DIS & Walt Disney & XOM & Exxon Mobil \\
DD & EI DuPont de Nemours & KO & Coca Cola \\
GE & General Electric & F & Ford \\
GM & General Motors & IBM & International Business Machines \\
HAL & Halliburton & JNJ & Johnson \& Johnson \\
MER & Merrill Lynch & MCD & MacDonald's \\
BAC & Bank of America & MMM & 3M Company \\
PFE & Pfizer Inc. & MCK & Mckesson \\
JPM & J. P. Morgan Chase & RTN & Raytheon \\
HPQ & Hewlett-Packard & UTX & United Technologies \\
HON & Honeywell International Inc. & &
\end{tabular}




\section{References}

[1] T.W. Anderson. An Introduction to Multivariate Statistical Analysis. New York, John Willey, 3rd edition, 2003. 3.2

[2] S. Benartzi, and R. Thaler. Illusionary Diversification and Its Implications for the U.S. and Chilean Retirement Systems. Working Paper, University of California at Los Angeles, 1998. 5.1

[3] P. Billingsley. Probability and Measure. New York: J.Wiley \& Sons, 3rd edition, 1995. 12

[4] N.F. Chen., R. Roll and S.A. Ross. Economic Forces and the Stock Market. The Journal of Business, 59:383-403, 1986. 4.1

[5] J.H. Cochrane. A Cross-Sectional Test of an Investment-Based Asset Pricing Model. The Journal of Political Economy, 104(3):572-621, 1996. 4.1

[6] J.H. Cochrane. Asset Pricing. Princeton, 2001. 4.1

[7] M.A.H. Dempster, I. V. Evstigneev, and K. R. Schenk-Hoppé, Volatility-Induced Financial Growth, Quantitative Finance, 7(2):151-160, 2007. 1

[8] I.V. Evstigneev, T. Hens and K.R. Schenk-Hoppé. Market selection of Financial Trading Strategies: Global Stability, Mathematical Finance, 12:329-339, 2002. 2, 2, 5.4

[9] I.V. Evstigneev, T. Hens and K.R. Schenk-Hoppé. Evolutionary Stable Stock Markets, Economic Theory, 27:449-468, 2006. 2, 2, 2, 7, 2, 2, 5.4

[10] E. Fama and K. French. Common Risk Factors in the Returns on Bonds and Stocks. Journal of Financial Economics, 33:3-56, 1993. 4.1

[11] R.Fernholz. Stochastic Portfolio Theory. Springer-Verlag, 2002. 1

[12] R. Fernholz and C. Maguire. The statistics of 'statistical arbitrage' in stock markets. Financial Analysts Journal, 63(5):46-52, 2007. 1, 5.1, 5.2

[13] R. Geske. The Pricing of Options with Stochastic Dividend Yield. The Journal of Finance, 33(2):617625, 1978. 3.1

[14] T. Hens, K.R. Schenk-Hoppé and M. Stalder. An Application of Evolutionary Finance to Firms Listed in the Swiss Market Index. Swiss Journal of Economics and Statistics, 138:465-487, 2002. 1, 2, 5.1, 5.3, $24,5.4,5.4$

[15] T. Hens, K.R. Schenk-Hoppé. Survival of the Fittest on Wall Street. Institute for Empirical Research in Economics, University of Zurich, 2004. 1, 2, 5.1, 5.3, 5.4, 27

[16] T. Hens, K.R. Schenk-Hoppé. Evolutionary Stability of Portfolio Rules in Incomplete Markets. Journal of Mathematical Economics, 41:43-66, 2005. 2, 5.3, 5.4, 5.4

[17] R. A. Johnson and D.W. Wichern. Applied Multivariate Statistical Analysis, 2nd Edition, 1988. 4.1, 17

[18] I.T. Jolliffe. Principal Component Analysis. Springer Statistics, 2nd edition, 2002. 3.4

[19] D.G. Luenberger. Investment Science. New York: Oxford University Press, 1997. 1, 8

[20] T. Marsh and R. Merton. Dividend variability and Variance bounds tests for the rationality of Stock Market Prices. The American Review, 76(3):483-498, 1986. 1, 9, 26

[21] K. Mavroudis. Constant Proportions Strategies in an Evolutionary Context Under a Dividend Factor Model. PhD Dissertation, Florida State University, 2008. 2, 3.2, 28

[22] R. Shiller. Do Stock Prices Move Too Much to be Justified by Subsequent Changes in Dividends? The American Economic Review, 71(3):421-436, 1981. 4.2, 5.3, 5.4 
[23] M.J. Smith, and G. Price. The logic of Animal Conflict. Nature, 246:15-18, 1973. 1

[24] M.J. Smith. The Theory of Games and the Evolution of Animal Conflicts. Journal of Theoretical Biology, 47:209-219, 1974. 